\title{
HST, VLT, and NTT imaging search for wide companions to bona-fide and candidate brown dwarfs in the Cha I dark cloud $^{\star}$
}

\author{
R. Neuhäuser ${ }^{1}$, W. Brandner ${ }^{2}$, J. Alves $^{2}$, V. Joergens ${ }^{1}$, and F. Comerón ${ }^{2}$ \\ 1 MPI für extraterrestrische Physik, Giessenbachstraße 1, 85740 Garching, Germany \\ 2 European Southern Observatory, Karl-Schwarzschild-Straße 2, 85748 Garching, Germany
}

Received 6 November 2001 / Accepted 14 January 2002

\begin{abstract}
We present results from a deep imaging search for companions around the young bona-fide and candidate brown dwarfs Cha $\mathrm{H} \alpha 1$ to 12 in the Cha I dark cloud, performed with HST WFPC2 $(R, I, \mathrm{H} \alpha)$, VLT FORS1 $(V R I)$, and NTT SofI $\left(J H K_{\mathrm{s}}\right)$. We find 16 faint companion candidates around five primaries with separations between $1.5^{\prime \prime}$ and $7^{\prime \prime}$ and magnitudes in $R \& I$ from 19 to 25 mag, i.e. up to 8 mag fainter than the primaries. While most of these companion candidates are probably unrelated background objects, there is one promising candidate, namely $1.5^{\prime \prime} \mathrm{SW}$ off the M6-dwarf Cha $\mathrm{H} \alpha$ 5. This candidate is 3.8 to 4.7 mag fainter than the primary and its colors are consistent with an early- to mid-L spectral type. Assuming the same distance $(140 \mathrm{pc})$ and absorption $\left(A_{I}=0.47 \mathrm{mag}\right)$ as towards the primary, the companion candidate has $\log \left(L_{\mathrm{bol}} / L_{\odot}\right)=-3.0 \pm 0.3$. At the age of the primary (1 to 5 Myrs), the faint object would have a mass of 3 to 15 Jupiter masses according to Burrows et al. (1997) and Chabrier \& Baraffe (2000) models. The probability for this companion candidate to be an unrelated fore- or background object is $\leq 0.7 \%$, its colors are marginally consistent with a strongly reddened background $\mathrm{K}$ giant. One other companion candidate has infrared colors consistent with an early T-dwarf. In addition, we present indications for Cha $\mathrm{H} \alpha 2$ being a close $\left(\sim 0.2^{\prime \prime}\right)$ binary with both components very close to the sub-stellar limit. Our detection limits are such that we should have detected all companions above $\sim 1 M_{\text {jup }}$ with separations $\geq 2^{\prime \prime}(\geq 320 \mathrm{AU})$ and all above $\sim 5 M_{\text {jup }}$ at $\geq 0.35^{\prime \prime}$ ( $\left.\geq 50 \mathrm{AU}\right)$.
\end{abstract}

Key words. stars: binaries: visual - individual: Cha H $\alpha 1$ to 12 - low-mass, brown dwarfs - pre-main sequence

\section{Introduction: Multiplicity of brown dwarfs}

Extrasolar planets were detected by indirect methods, but no direct imaging was presented, yet. Imaging of sub-stellar companions around young very low-mass stars or brown dwarfs (rather than around normal solar-type stars) should be less difficult, because young sub-stellar objects are self-luminous due to ongoing contraction and very low-mass stars and brown dwarfs as primaries are intrinsically faint. Hence, the problem of dynamic range (large magnitude difference at small angular separation) is not that severe. Only a few brown dwarfs were detected directly as companions to normal stars so far, mostly around M-dwarfs, the first of which was Gl 229 B (Nakajima et al. 1995, Oppenheimer et al. 1995), and the youngest of which is TWA-5 B (Lowrance et al. 1999; Neuhäuser et al. 2000).

Send offprint requests to: R. Neuhäuser,

e-mail: rne@mpe.mpg.de

* Based on observations obtained with the NASA/ESA Hubble Space Telescope in program GO 8716, ESO programs 64.I-0493, 66.C-0022, and 66.C-0310 as well as data taken from the public VLT data archive.
Brandner et al. (2000) undertook an HST and AO survey for sub-stellar companions around T Tauri stars, which we now extend to the M6- to M8-type objects Cha $\mathrm{H} \alpha 1$ to 12 in the Cha I dark cloud. We obtained $\mathrm{H} \alpha$ as well as $R$ - and $I$-band images with the Hubble Space Telescope (HST) in order to search for wide visual companions around them. These observations are both deeper and have higher spatial resolution than any previous images of these objects. We complement the HST data with archived $V R I$ data from the ESO $8.2 \mathrm{~m}$ Very Large Telescope (VLT) and new $J H K_{\mathrm{s}}$ data from the ESO $3.5 \mathrm{~m}$ New Technology Telescope (NTT).

With deep imaging of bona-fide and candidate brown dwarfs as presented here, one can, in principle, find binaries comprised of (i) two low-mass stars (as some of the candidate brown dwarfs in the sample could be very lowmass stars), (ii) one brown dwarf and one low-mass star, (iii) two brown dwarfs, (iv) one giant planet and one lowmass star, (v) or one giant planet and one brown dwarf.

So far, only little is known about multiplicity of brown dwarfs: The first binary brown dwarf found was 
PPl 15, a spectroscopic binary made up of two brown dwarfs in the Pleiades (Basri \& Martín 1999). Then, faint companion candidates were detected directly near a few brown dwarfs, namely DENISJ1228.2 (Martín et al. 1999), 2MASSWJ1146 (Koerner et al. 1999), and 2MASSJ0850 (Reid et al. 2001).

Multiplicity parameters of brown dwarfs (like binary frequency, orbit characteristics, mass functions of primaries and secondaries, etc.) will shed light on their as yet uncertain formation mechanism: Do brown dwarf companions form like stellar companions or like planets in circumstellar disks? Do they form by fragmentation or core growth? Do isolated brown dwarfs form in isolation or are they ejected from multiple systems? If brown dwarfs are ejected in the early accretion phase (Reipurth \& Clarke 2000 ), then one should not find any brown dwarfs as companions at ages $\geq 1$ Myrs. If low-mass companions are ejected during the pre-main sequence phase (cf. Sterzik et al. 2001), then young brown dwarf companions should be more frequent than old brown dwarf companions, which we can test with our sample of young objects in Cha I.

It is as yet unknown, whether brown dwarfs can have planets and, if so, what their typical separations from the primary objects would be. With several high-resolution spectra obtained with VLT/UVES, Joergens \& Guenther (2001) found evidence for radial velocity variations in some of the Cha $\mathrm{H} \alpha$ objects, which could be due to giant planets or surface features.

In Sect. 2, we will present the properties of the targets. In Sect. 3, details about the HST observations and data reduction are given. The complementary VLT and NTT observations are presented in Sect. 4. The background contamination is estimated in Sect. 5. Properties of the most promising companion candidate are discussed in Sect. 6. In Sect. 7, we present indications for one of the primaries being a very close $\sim 0.2^{\prime \prime}$ binary pair. We conclude with a brief discussion in Sect. 8 .

\section{Our targets: Cha $\mathrm{H} \alpha \mathbf{1}$ to 12}

The Cha I dark cloud is a site of ongoing low- and intermediate mass star formation at $\sim 140$ to 160 pc. Most of the known members are pre-main sequence $\mathrm{T}$ Tauri stars, but there is also one known intermediate-mass Herbig A0e star (HD 97048) and several M6- to M8-type bona-fide and candidate brown dwarfs. The latter objects (Cha $\mathrm{H} \alpha 1$ to 12) were found in deep infrared (IR), $\mathrm{H} \alpha$, and X-ray surveys (Neuhäuser \& Comerón 1998, 1999; Comerón et al. 1999, 2000, henceforth C1999 \& C2000), e.g. Cha H $\alpha 1$ was the first X-ray detected brown dwarf. With ground-based optical and near-IR data as well as mid-IR data from ISO (C2000, Persi et al. 2000), Natta \& Testi (2001) show that Cha $\mathrm{H} \alpha 1,2$, and 9 have IR excess emission indicative for circumstellar material like disks. Also, Wilking et al. (1999) and Muench et al. (2001) found evidence for circumstellar material around young brown dwarfs in $\rho$ Oph and the Trapezium, respectively.
Based on their location in the $H-R$ diagram and comparison with different theoretical isochrones, Cha $\mathrm{H} \alpha 1$ to 12 are roughly co-eval, namely $\sim 2$ Myrs according to Baraffe et al. (1998) and $\sim 1$ to 10 Myrs according to Burrows et al. (1997). The M7.5-M8 type objects Cha $\mathrm{H} \alpha 1,7,10$, and 11 are clearly located below the sub-stellar limit and, hence, were classified as bona-fide brown dwarfs, while the other Cha $\mathrm{H} \alpha$ objects are candidate brown dwarfs (C2000). The brightest of them, Cha $\mathrm{H} \alpha 1$ to 8 and 12, are known to show Lithium absorption and radial velocity consistent with membership to the Cha I T association (Neuhäuser \& Comerón 1999; Joergens \& Guenther 2001), the others were not observed with high resolution, yet, because of their faintness. Previously, none of the bona-fide and candidate brown dwarfs Cha $\mathrm{H} \alpha 1$ to 12 observed here were known to be multiple.

\section{HST observation and data reduction}

In program 8716, we obtained deep imaging with the HST Wide Field Planetary Camera No. 2 (WFPC2) filters F656N (H $\alpha)$, F675W ( $R$-band), and F814W ( $I$-band). We performed the HST observations in the optical (rather than in the IR), because NICMOS was not available during the last HST cycle. For our WFPC observations, the two reddest broad-band filters were used ( $R \& I$, because substellar objects are red) as well as the $\mathrm{H} \alpha$ filter; the latter was used, because all the primary objects Cha $\mathrm{H} \alpha 1$ to 12 show $\mathrm{H} \alpha$ emission, so that any additional faint object with $\mathrm{H} \alpha$ emission would be a good candidate for a hithertoo unknown brown dwarf.

Faint companions to any of the targets would be substellar, because all our (as yet unresolved) targets are located either below or very close to the sub-stellar limit, so that any much fainter, i.e. lower-mass companion would certainly be sub-stellar.

Because of missing guide stars (all targets are located inside the dark cloud Cha I), Cha $\mathrm{H} \alpha 6$ could not be observed. All other eleven targets were observed.

Whenever possible, we placed several objects in the field-of-view of the WFPC chips to maximize the efficiency. Isolated objects were placed onto the PC chip for better angular resolution: The PC chip has a $0.0455^{\prime \prime} /$ pixel scale, the WF chips have a scale of $0.0996^{\prime \prime} /$ pixel. In total, we observed in eight HST orbits in 2000 and 2001. See Table 1 for the HST observation log.

Data reduction was performed with the $\mathrm{IRAF}^{1}$ package stsdas written for HST data. All exposures (for each field and each filter) were split into two to four to facilitate for cosmic ray rejection. After rejection of cosmic rays with crrej, we co-added the data. Mostly, the background was very small ( $\sim 1$ count), because the targets are in the dark

\footnotetext{
${ }^{1}$ IRAF is distributed by the National Optical Astronomy Observatories, which is operated by the Association of Universities for Research in Astronomy, Inc. (AURA) under cooperative agreement with the National Science Foundation.
} 
Table 1. Observing log of HST WFPC2 observations (*).

\begin{tabular}{|c|c|c|c|}
\hline $\begin{array}{l}\text { Object } \\
\text { in Cha I }\end{array}$ & filter & $\begin{array}{l}\text { exposure } \\
\text { [seconds] }\end{array}$ & $\begin{array}{l}\text { JD } \\
\text { date }\end{array}$ \\
\hline \multirow[t]{3}{*}{$\mathrm{H} \alpha 1 \& 7$} & F656N (H $\alpha)$ & $2 \times 500$ & 2451952 \\
\hline & F675W (R) & $2 \times 100$ & 12 Feb. 2001 \\
\hline & F814W (I) & $2 \times 100$ & \\
\hline \multirow[t]{3}{*}{$\mathrm{H} \alpha 2 \& 9$} & $\mathrm{~F} 656 \mathrm{~N}(\mathrm{H} \alpha)$ & $2 \times 500$ & 2451842 \\
\hline & $\mathrm{F} 675 \mathrm{~W}(\mathrm{R})$ & $2 \times 100$ & 25 Oct. 2000 \\
\hline & F814W (I) & $2 \times 100(* *)$ & \\
\hline \multirow[t]{3}{*}{$\mathrm{H} \alpha 3$} & $\mathrm{~F} 656 \mathrm{~N}(\mathrm{H} \alpha)$ & $2 \times 700$ & 2451733 \\
\hline & $\mathrm{F} 675 \mathrm{~W}(\mathrm{R})$ & $300+80$ & 8 Jul. 2000 \\
\hline & F814W (I) & $160+40$ & \\
\hline $\mathrm{H} \alpha 4,10,11$ & $\mathrm{~F} 656 \mathrm{~N}(\mathrm{H} \alpha)$ & $4 \times 500$ & 2451783 \\
\hline \multirow[t]{2}{*}{$\& 8(* * *)$} & F675W (R) & $2 \times(300+100)$ & 27 Aug. 2000 \\
\hline & F814W (I) & $2 \times(300+100)$ & \\
\hline \multirow[t]{3}{*}{$\mathrm{H} \alpha 5$} & $\mathrm{~F} 656 \mathrm{~N}(\mathrm{H} \alpha)$ & $2 \times 700$ & 2451778 \\
\hline & F675W (R) & $300+80$ & 22 Aug. 2000 \\
\hline & F814W (I) & $160+40$ & \\
\hline \multirow[t]{3}{*}{$\mathrm{H} \alpha 8$} & $\mathrm{~F} 656 \mathrm{~N}(\mathrm{H} \alpha)$ & $2 \times 700$ & 2451752 \\
\hline & $\mathrm{F} 675 \mathrm{~W}(\mathrm{R})$ & $300+80$ & 27 Jul. 2000 \\
\hline & F814W (I) & $160+40$ & \\
\hline \multirow[t]{3}{*}{$\mathrm{H} \alpha 12$} & $\mathrm{~F} 656 \mathrm{~N}(\mathrm{H} \alpha)$ & $2 \times(260+70)$ & 2451914 \\
\hline & $\mathrm{F} 675 \mathrm{~W}(\mathrm{R})$ & $2 \times(10+40)$ & 5 Jan. 2001 \\
\hline & F814W (I) & $2 \times(10+40)$ & \\
\hline
\end{tabular}

Remarks: $\left(^{*}\right)$ There are no other (archived or scheduled) HST observations with any of the targets in the field-of-view (as of Sep. 2001). (**) The observations in the $I$ filter failed due to technical problems, hence no data in $I$. (***) Cha $\mathrm{H} \alpha 8$ and a few of its companion candidates were again in the field of these observations, namely at the edge of chip WF2.

cloud Cha I, while the background was slightly higher near the bright $(V=8.5 \mathrm{mag})$ A0e star HD 97048 .

Source detection was done by visual inspection. A faint, non-extended object near any of the targets, i.e. within one or a few arcsec, can in principle be either a real companion or an unrelated fore- or background object. Since we have no information, yet, on the spectra or proper motions of these faint objects, all of them have to be regarded as companion candidates, unless companionship can already be ruled out from color or extension. For the maximum separation between a primary target and a potential companion, we allow for (more or less arbitrarily) $1000 \mathrm{AU}$, which is $\sim 7^{\prime \prime}$ at the distance of Cha I $(\sim 140$ to $160 \mathrm{pc}$ ). Hence, in Table 2, we list only those faint objects, which are found within $7^{\prime \prime}$ around the $\mathrm{Cha} \mathrm{H} \alpha$ targets, while other faint objects are regarded as unrelated objects most likely located in the background of Cha I. The observed background population is used in Sect. 5 for a rough estimation as to how likely the companion candidates are true companions rather than unrelated objects.

In Figs. 1 and 2, we show the HST I-band images of those Cha $\mathrm{H} \alpha$ objects, where several companion candidates were detected within $7^{\prime \prime}$, namely $\mathrm{Cha} \mathrm{H} \alpha 4$, 10, \& 11 (Fig. 1) and Cha $\mathrm{H} \alpha 8$ (Fig. 2), while Cha $\mathrm{H} \alpha 5$ is shown in Figs. 8 to 10. The faint companion candidates are labeled with their cc numbers given in Table 2 (cc for

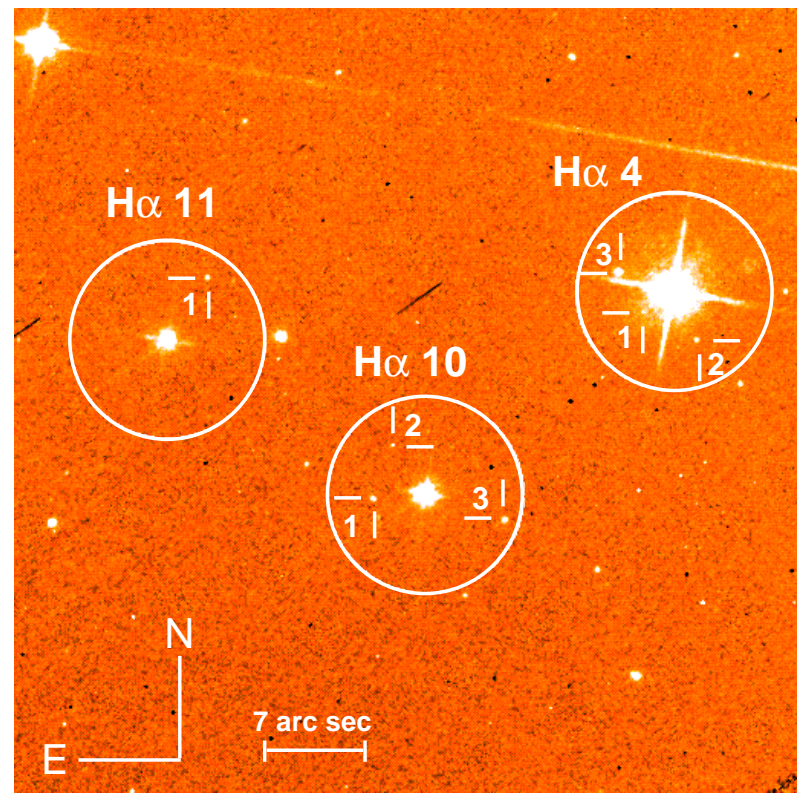

Fig. 1. HST I-band image of Cha $\mathrm{H} \alpha 4,10$, and 11 and surrounding field, exposure time is $800 \mathrm{~s}$, obtained on 27 Aug. 2000. The circles around the three main targets each have $7^{\prime \prime}$ radii, located on the WF4 chip.

companion candidate). The companion candidates Cha $\mathrm{H} \alpha 8 /$ cc $1 \& 2$ are detected only in the observation which mainly aimed at $\mathrm{Cha} \mathrm{H} \alpha 4,10$, and 11, where Cha $\mathrm{H} \alpha 8$ was located at the edge of chip WF2. Because one of the main targets, Cha $\mathrm{H} \alpha 6$, was not observable due to the lack of guide stars, we assigned the extra observing time (one more orbit) for the observation with Cha $\mathrm{H} \alpha 4,10, \& 11$ on the chip WF4, because of the large number of primaries observable simultaneously. Only due to the increased exposure time (800 s compared to $200 \mathrm{~s}$ in the $I$-band), the faint companion candidates Cha $\mathrm{H} \alpha 8 /$ cc $1 \& 2$ were detected. They are detected only in $I$, see Fig. 2. Due to the longer exposure time in this observation, Cha $\mathrm{H} \alpha 4$ got saturated in $I$.

Aperture photometry was obtained for all objects (detected by visual inspection) with a $0.5^{\prime \prime}$ radius with a source-free background field annulus located around the measured object. The counts were transformed to magnitudes $m$ using

$m[\mathrm{mag}]=-2.5 \cdot \log (D N / \exp )+Z P-0.1$

with the number of counts $D N$, the exposure time $\exp$ in seconds, and the zero point $Z P$; the subtraction of $0.1 \mathrm{mag}$ is to correct from the $0.5^{\prime \prime}$ radius to the infinite aperture; all according to the HST data reduction manual ${ }^{2}$.

Magnitudes obtained with Eq. (1) have still to be corrected for the charge-transfer-efficiency (CTE) loss. This was done according to Whitmore et al. (1999) with the number of counts, the observational date, the background level, and the $(x, y)$ pixel position of the source peak as inputs. The CTE corrections are especially important for

\footnotetext{
${ }^{2}$ http://www.stsci.edu/documents/dhb/web
} 

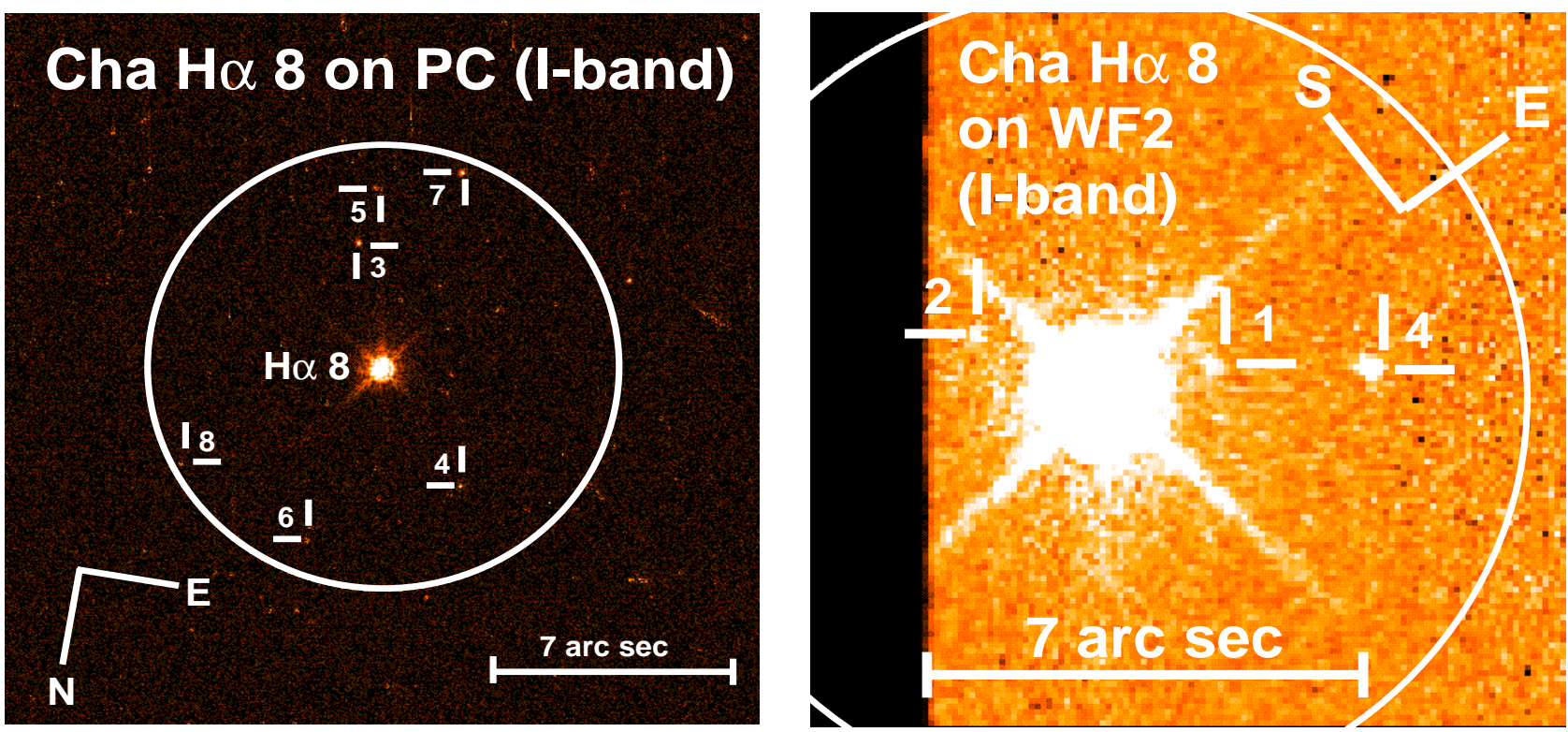

Fig. 2. HST I-band images of Cha $\mathrm{H} \alpha 8$ and surrounding field: The left image shows the $200 \mathrm{~s}$ integration on the PC chip (obtained on 27 July 2000), the right panel shows the $800 \mathrm{~s}$ integration (27 Aug. 2000), where the target is located at the edge of the chip WF2, where two more faint companion candidates were detected. The circles have $7^{\prime \prime}$ radii.

weak sources such as the brown dwarf companion candidates expected in our program. The final values are given in Table 3.

A comparison of the HST $\mathrm{H} \alpha$ magnitudes with previously known gound-based VLT $\mathrm{H} \alpha$ equivelent widths (C2000) shows a clear trend (see Fig. 3), with a scatter indicating strong $\mathrm{H} \alpha$ variability, not unexpected for such young objects. In Fig. 3, we plot the ground based $\mathrm{H} \alpha$ equivalent width versus the difference between $\mathrm{HST} \mathrm{H} \alpha$ and $R$-band magnitude, to really compare the $\mathrm{H} \alpha$ line flux above the continuum with the equivalent width.

\section{Ground-based imaging with VLT and NTT}

We also used archived VRI data ${ }^{3}$ obtained with the FOcal Reducer/low dispersion Spectrograph No. 1 (FORS1) at the ESO $8.2 \mathrm{~m}$ telescope Antu, Unit Telescope 1 (UT1) of the VLT, with a scale of $0.2^{\prime \prime} /$ pixel, during sub-arcsec conditions. See Table 4 for the observations log.

We re-reduced the ground-based images. Some companion candidates were also detected in the VLT VRI images, magnitudes are given in Table 3. Comparison of the HST $R \& I$ magnitudes (Vega system) for all the Cha $\mathrm{H} \alpha 1$ to 12 objects with the previously known ground-based $R$ \& I magnitudes (Cousins bands) from C2000 shows that they agree well $( \pm 0.1 \mathrm{mag})$.

IR images in $J H K_{\mathrm{s}}$ were obtained using the IR imager Son of Isaac (SofI) at the ESO $3.5 \mathrm{~m}$ NTT on La Silla with $\sim 0.6$ to $0.7^{\prime \prime}$ seeing. Images of three fields were taken in March 2001 with the small SofI field to achieve the

\footnotetext{
${ }^{3}$ Obtained after commissioning of FORS1 before the start of normal operations; the data can be retrieved from the public VLT data archive; see C2000 for details, where the same data were used to estimate the $V R I$ values of Cha $\mathrm{H} \alpha 1$ to 12 .
}

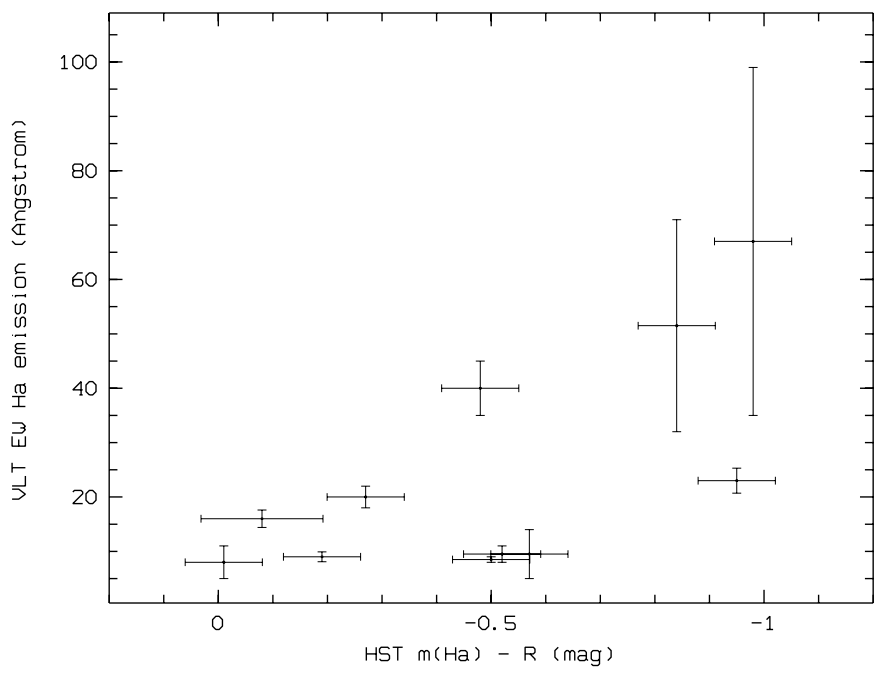

Fig. 3. Ground-based $\mathrm{H} \alpha$ equivalent width (with its observed range due to variability or an assumed $10 \%$ error bar) versus HST H $\alpha$ measurement (plotted as difference between HST Vega $\mathrm{H} \alpha$ magnitude and HST Vega $R$-band magnitude, so that we plot the line strength above continuum as for the equivalent width) for the Cha $\mathrm{H} \alpha$ primaries (none of the companion candidates were detected in $\mathrm{H} \alpha$ ), data from Table 3. There is a trend for a correlation: The brighter the HST $\mathrm{H} \alpha$ magnitude (after subtraction of $R$ ), the larger the ground-based $\mathrm{H} \alpha$ equivalent width, as expected, with some scatter most certainly due to variability, as expected.

highest angular resolution possible $\left(0.147^{\prime \prime} /\right.$ pixel $)$ with $2 \mathrm{~s}$ individual exposure per frame. Two more images $(H \&$ $K_{\mathrm{S}}$ ) were already taken in March 2000 with the large SofI field $\left(0.29^{\prime \prime} /\right.$ pixel $)$, so that several Cha $\mathrm{H} \alpha$ primaries were in the field-of-view, with 3 s individual exposure per frame. Darks, flats, and standards were taken in the same nights, 
Table 2. List of all wide companion candidates found in the HST imaging data. The object designations contain the J2000.0 positions. In the remainder of the paper, we use the other names, where the designation $c c$ means companion candidate. The offsets (typically better than $\pm 0.1^{\prime \prime}$ ) of the companion candidates (sorted by increasing separations up to $7^{\prime \prime}$, the upper limit) are given with respect to the primary. Spectral types for the primaries are from C2000.

\begin{tabular}{|c|c|c|c|c|}
\hline \multirow{2}{*}{$\begin{array}{l}\text { Object } \\
\text { designation }\end{array}$} & \multirow[t]{2}{*}{ other name } & \multirow{2}{*}{$\begin{array}{l}\text { HST } \\
\text { chip }\end{array}$} & \multicolumn{2}{|c|}{ separation ["'] } \\
\hline & & & $\Delta \alpha$ & $\Delta \delta$ \\
\hline $110717.0-773454$ & Cha $\mathrm{H} \alpha 1$ & WF4 & primary & M7.5 \\
\hline 110743.0-773359 & Cha $\mathrm{H} \alpha 2$ & WF4 & primary & M6.5 \\
\hline $110752.9-773656$ & Cha $\mathrm{H} \alpha 3$ & $\mathrm{PC}$ & primary & M7 \\
\hline 110819.6-773917 & Cha $\mathrm{H} \alpha 4$ & WF4 & primary & M6 \\
\hline 110819.7-773918 & $\mathrm{H} \alpha 4 / \operatorname{cc~} 1$ & WF4 & $2.0 \mathrm{E}$ & $1.3 \mathrm{~S}$ \\
\hline 110819.5-773920 & $\mathrm{H} \alpha 4 / \mathrm{cc} 2$ & WF4 & $1.4 \mathrm{~W}$ & $3.3 \mathrm{~S}$ \\
\hline 110819.8-773918 & $\mathrm{H} \alpha 4 / \mathrm{cc} 3$ & WF4 & $3.5 \mathrm{E}$ & $1.3 \mathrm{~N}$ \\
\hline $110825.6-774146$ & Cha $\mathrm{H} \alpha 5$ & $\mathrm{PC}$ & primary & M6 \\
\hline $110825.5-774147$ & $\mathrm{H} \alpha 5 / \mathrm{cc} 1$ & $\mathrm{PC}$ & $1.0 \mathrm{~W}$ & $1.1 \mathrm{~S}$ \\
\hline $110840.2-773417$ & Cha $\mathrm{H} \alpha 6$ & \multicolumn{2}{|c|}{ not observed } & M7 \\
\hline $110738.4-773530$ & Cha $\mathrm{H} \alpha 7$ & WF2 & primary & M8 \\
\hline $110747.8-774008$ & Cha $\mathrm{H} \alpha 8$ & $\mathrm{PC}$ & primary & M6.5 \\
\hline 110747.9-774007 & Н $\alpha$ 8/cc 1 & WF2 & $1.4 \mathrm{E}$ & $1.3 \mathrm{~N}$ \\
\hline $110747.8-774010$ & Н $\alpha 8 /$ cc 2 & WF2 & $0.4 \mathrm{~W}$ & $2.3 \mathrm{~S}$ \\
\hline $110747.7-774012$ & $\mathrm{H} \alpha 8 / \operatorname{cc~} 3$ & $\mathrm{PC}$ & $1.3 \mathrm{~W}$ & $4.0 \mathrm{~S}$ \\
\hline 110748.0-774004 & $\mathrm{H} \alpha 8 / \operatorname{cc~} 4$ & $\mathrm{PC}$ & $2.9 \mathrm{E}$ & $3.5 \mathrm{~N}$ \\
\hline $110747.7-774014$ & $\mathrm{H} \alpha 8 / \operatorname{cc} 5$ & $\mathrm{PC}$ & $0.8 \mathrm{~W}$ & $5.8 \mathrm{~S}$ \\
\hline $110747.6-774003$ & Н $\alpha 8 / \operatorname{cc} 6$ & $\mathrm{PC}$ & $3.0 \mathrm{~W}$ & $5.3 \mathrm{~N}$ \\
\hline 110747.9-774015 & $\mathrm{H} \alpha 8 / \operatorname{cc~} 7$ & $\mathrm{PC}$ & $1.7 \mathrm{E}$ & $6.7 \mathrm{~S}$ \\
\hline 110747.4-774006 & $\mathrm{H} \alpha 8 / \operatorname{cc~} 8$ & $\mathrm{PC}$ & $6.6 \mathrm{~W}$ & $2.4 \mathrm{~N}$ \\
\hline $110719.2-773252$ & Cha $\mathrm{H} \alpha 9$ & WF2 & primary & M6 \\
\hline $110825.6-773930$ & Cha $\mathrm{H} \alpha 10$ & WF4 & primary & M7.5 \\
\hline $110825.8-773930$ & $\mathrm{H} \alpha 10 /$ cc 1 & WF4 & $3.4 \mathrm{E}$ & $0.3 \mathrm{~S}$ \\
\hline 110825.7-773927 & H $\alpha 10 /$ cc 2 & WF4 & $2.2 \mathrm{E}$ & $3.2 \mathrm{~N}$ \\
\hline $110825.3-773932$ & $\mathrm{H} \alpha 10 /$ cc 3 & WF4 & $5.2 \mathrm{~W}$ & $1.7 \mathrm{~S}$ \\
\hline $110830.8-773919$ & Cha $\mathrm{H} \alpha 11$ & WF4 & primary & M8 \\
\hline 110830.6-773915 & $\mathrm{H} \alpha 11 / \mathrm{cc} 1$ & WF4 & $2.7 \mathrm{~W}$ & $4.0 \mathrm{~N}$ \\
\hline $110637.4-774307$ & Cha $\mathrm{H} \alpha 12$ & WF4 & primary & M7 \\
\hline
\end{tabular}

and we performed standard data reduction with eclipse ${ }^{4}$ and $M I D A S^{5}$. See Table 4 for the observations $\log$ and Table 3 for the $J H K_{\mathrm{s}}$ magnitudes. Our $J H K_{\mathrm{s}}$ data for the primaries (Table 3) agree well with those given in C2000, who had obtained their data with IRAC2 at the $\mathrm{ESO} / \mathrm{MPG} 2.2 \mathrm{~m}$.

The new $I J K_{\mathrm{s}}$ data listed in Table 3 for the primaries also agree well with the DENIS magnitudes listed in Neuhäuser \& Comerón (1999) for Cha H $\alpha 1$ to 8. The $H$-band magnitude of Cha $\mathrm{H} \alpha 8$ (Table 3 ) was not known before, as this object was not observed in the IR by C2000 and as the $H$-band filter is not used for DENIS. The $J$ $\& K_{\mathrm{s}}$ magnitudes for Cha $\mathrm{H} \alpha 8$ given in Table 3 (from the March 2000 image) agree with the DENIS magnitudes given in Neuhäuser \& Comerón (1999).

Most of our ground-based $V R I J H K_{\mathrm{s}}$ magnitudes were determined by normal aperture photometry, with the

\footnotetext{
4 see www.eso.org/projects/aot/eclipse/

5 see www.eso.org/projects/esomidas/
}

exception for the $V R I J$-band images for the very close companion candidate near $\mathrm{Cha} \mathrm{H} \alpha 5$, which is discussed in detail in Sect. 6.

Lower limits to the magnitudes for undetected objects are derived from mean background intensity (and its variation) from \pm 4 pixels (for HST images) and \pm 3 pixels (for ground-based images) around the pixel, where the peak of the companion candidate should be located according to its position. The limits given correspond to an intensity of $3 \sigma$ above the mean background. No additonal companion candidates (within $7^{\prime \prime}$ ) were detected in the groundbased images.

\section{Real companions or background objects?}

A first check, whether or not a companion candidate may be truely bound (hence at the same distance and age as primary), i.e. whether or not it is as cool as expected from the magnitude difference, can be done with the optical and IR colors. For several companion candidates, one can find a solution for the observed magnitudes and colors for either a moderately reddened L- or T-type companion or, alternatively, for a highly reddened background star. In the next section, we go through such an estimate for the companion candidate near Cha $\mathrm{H} \alpha 5$.

Figures 4 and 5 show that most companion candidates have colors similar to background giants, while some of them have colors which are (also) consistent with Ldwarfs. Of those, Cha $\mathrm{H} \alpha 5 / \mathrm{cc} 1$ has the smallest separation and is therefore the best companion candidate (see next section for a detailed discussion of this object). Given their faint magnitudes, all these companion candidates would have masses around or below the deuterium burning limit. For such low masses, they should be very faint and red in the optical. However, with the possible exception of Cha $\mathrm{H} \alpha 5 / \mathrm{cc} 1$ and Cha $\mathrm{H} \alpha 4 / \mathrm{cc} 2$, they are all too bright in $R \& I$ given their $J H K_{\mathrm{s}}$ values, so that they are probably reddened background giants or extragalactic. Cha $\mathrm{H} \alpha 8 / \mathrm{cc} 7$ is located in the area of IR excess (Fig. 5), possibly indicating circumstellar material.

Another possibly interesting object is Cha $\mathrm{H} \alpha 4 / \mathrm{cc} 2$ with $J-H=1.10 \pm 0.14$ and $H-K_{\mathrm{s}}=0.10 \pm$ $0.14 \mathrm{mag}$, the bluest in $H-K_{\mathrm{s}}$ in Fig. 5. The T3-dwarf SDSSpJ102109.69-030420.1 has similar (2MASS) colors according to Burgasser et al. (2001), namely $J-H=$ $0.93 \pm 0.15$ and $H-K_{\mathrm{s}}=0.23 \pm 0.20 \mathrm{mag}^{6}$. If this object would really be an early T-dwarf companion, it would definitely be a planetary mass-object. Its separation, however, is $3.6^{\prime \prime}$, i.e. $\sim 500 \mathrm{AU}$ at $140 \mathrm{pc}$, larger than expected for planets.

Whether a certain companion candidate really is a bound companion or not, can be checked by spectroscopy (whether the atmosphere is as cool as expected) or proper motion (whether primary and companion are co-moving). For some of the HST companion candidates detected also

\footnotetext{
${ }^{6}$ But somewhat different colors according to Leggett et al. (2002), namely $J-H \simeq 0.47$ and $H-K \simeq 0.15$ mag.
} 
Table 3. Ground-based $V, R_{\mathrm{C}}, I_{\mathrm{C}}$ from VLT/FORS1, space-based $R_{\mathrm{V}}, I_{\mathrm{V}}, m(\mathrm{H} \alpha)$ from HST/WFPC2, and ground-based $J, H, K_{\mathrm{s}}$ from NTT/SofI: The ground-based VRI data for the primary objects are from C2000, the $J H K_{\mathrm{s}}$ for Cha $\mathrm{H} \alpha 1,2,3$, 7 , and 9 are also from C2000, but for all other primaries and all companion candidates from our NTT data, the ground-based $V R I$ magnitudes for the companion candidates were determined by us from the C2000 VLT/FORS1 images. Typical precision of the magnitudes are \pm 0.05 for the bright primary objects and \pm 0.1 for the companion candidates, but \pm 0.2 mag or worse for objects fainter than $23 \mathrm{mag}$ as well as Cha $\mathrm{H} \alpha$ 5/cc 1 because of its small separation (see Sect. 6); n/o for not observed; see text for determination of lower magnitude limits. We list the observed range in H $\alpha$ equivalent width $W_{\lambda}$ from C1999, Neuhäuser \& Comerón (1999), and C2000, $\mathrm{H} \alpha$ always in emission.

\begin{tabular}{|c|c|c|c|c|c|c|c|c|c|c|}
\hline Object & $\begin{array}{r}V \\
(\mathrm{VLT})\end{array}$ & $\begin{array}{r}R_{\mathrm{C}} \\
(\mathrm{VLT})\end{array}$ & $\begin{array}{r}m(\mathrm{~F} 675 \mathrm{~W}) \\
\left(\mathrm{HST} \mathrm{R}_{\mathrm{V}}\right)\end{array}$ & $\begin{array}{r}I_{\mathrm{C}} \\
(\mathrm{VLT})\end{array}$ & $\begin{array}{r}m(\mathrm{~F} 814 \mathrm{~W}) \\
\left(\mathrm{HST} \mathrm{I}_{\mathrm{V}}\right)\end{array}$ & $J$ & $H$ & $K_{\mathrm{s}}$ & $\begin{array}{l}m(\mathrm{~F} 656 \mathrm{~N}) \\
(\mathrm{HST} \mathrm{H} \alpha)\end{array}$ & $\begin{array}{c}W_{\lambda}(\mathrm{H} \alpha) \\
{[\AA]}\end{array}$ \\
\hline Cha $\mathrm{H} \alpha 1$ & 21.0 & 18.7 & 18.96 & 16.17 & 16.16 & 13.55 & 12.78 & 12.28 & 17.72 & $\begin{array}{l}35-99 \\
5\end{array}$ \\
\hline Cha $\mathrm{H} \alpha 2$ & 19.8 & 17.60 & 17.42 & 15.08 & $\mathrm{n} / \mathrm{o}$ & 12.59 & 11.43 & 11.15 & 16.76 & $32-71$ \\
\hline Cha $\mathrm{H} \alpha 3$ & 19.51 & 17.38 & 17.27 & 14.89 & 14.97 & 12.46 & 11.64 & 11.11 & 16.81 & $5-14$ \\
\hline Cha $\mathrm{H} \alpha 4$ & 18.52 & 16.70 & 16.68 & 14.34 & sat. & 12.19 & 11.38 & 11.09 & 16.69 & $5-11$ \\
\hline $\mathrm{H} \alpha 4 / \mathrm{cc} 1$ & $\geq 22.5$ & $\geq 22.2$ & $\geq 23.6$ & $\geq 19.0$ & 23.0 & $\geq 18.4$ & $\geq 18.1$ & $\geq 17.3$ & & \\
\hline $\mathrm{H} \alpha 4 / \operatorname{cc~} 2$ & $\geq 22.8$ & $\geq 22.3$ & 24.1 & $\geq 20.7$ & 22.2 & 18.5 & 17.4 & 17.3 & & \\
\hline $\mathrm{H} \alpha 4 / \mathrm{cc} 3$ & 23.0 & 21.6 & 21.7 & 20.4 & 20.2 & 17.7 & 16.7 & 16.2 & & \\
\hline Cha $\mathrm{H} \alpha 5$ & 19.18 & 17.14 & 17.03 & 14.68 & 14.67 & 12.14 & 11.21 & 10.56 & 16.62 & $8-11$ \\
\hline $\mathrm{H} \alpha 5 / \mathrm{cc} 1$ & 23.1: & 21.1: & 21.22 & 19.0: & 19.20 & 16.8: & 15.0: & 14.4: & & \\
\hline Cha $\mathrm{H} \alpha 6$ & 19.75 & 17.60 & $\mathrm{n} / \mathrm{o}$ & 15.13 & $\mathrm{n} / \mathrm{o}$ & 12.43 & 11.61 & 11.09 & $\mathrm{n} / \mathrm{o}$ & $59-76$ \\
\hline Cha $\mathrm{H} \alpha 7$ & 22.2 & 19.5 & 19.69 & 16.86 & 16.78 & 13.89 & 13.00 & 12.51 & 19.02 & $35-45$ \\
\hline Cha $\mathrm{H} \alpha 8$ & 20.1 & 17.96 & 17.84 & 15.47 & 15.50 & 12.9 & 12.0 & 11.4 & 17.46 & $8-9$ \\
\hline $\mathrm{H} \alpha 8 / \mathrm{cc} 1$ & $\geq 23.2$ & $\geq 21.5$ & $\geq 23.5$ & $\geq 19.3$ & 23.4 & $\geq 17.5$ & $\geq 16.6$ & $\geq 14.7$ & & \\
\hline Н $\alpha 8 / \operatorname{cc~} 2$ & $\geq 23.6$ & $\geq 22.5$ & $\geq 23.5$ & $\geq 20.4$ & 23.6 & $\geq 17.0$ & $\geq 18.1$ & $\geq 16.6$ & & \\
\hline $\mathrm{H} \alpha 8 / \mathrm{cc} 3$ & $\geq 23.3$ & 23.2 & 22.7 & 21.3 & 21.3 & 18.8 & 16.7 & 15.8 & & \\
\hline $\mathrm{H} \alpha 8 / \mathrm{cc} 4$ & $\geq 23.3$ & 22.9 & 22.9 & 21.5 & 21.7 & 19.9 & 17.8 & 17.0 & & \\
\hline $\mathrm{H} \alpha 8 / \mathrm{cc} 5$ & $\geq 23.5$ & $\geq 23.2$ & $\geq 24.8$ & $\geq 22.0$ & 21.7 & $\geq 20.3$ & $\geq 20.1$ & $\geq 18.3$ & & \\
\hline $\mathrm{H} \alpha 8 / \mathrm{cc} 6$ & $\geq 23.4$ & $\geq 23.1$ & 23.1 & $\geq 21.9$ & 22.6 & $\geq 20.6$ & $\geq 20.0$ & $\geq 19.3$ & & \\
\hline $\mathrm{H} \alpha 8 / \operatorname{cc} 7$ & $\geq 23.5$ & 22.9 & 22.5 & 21.0 & 21.0 & 18.4 & 16.8 & 15.4 & & \\
\hline $\mathrm{H} \alpha 8 / \mathrm{cc} 8$ & $\geq 23.4$ & $\geq 23.1$ & $\geq 24.9$ & $\geq 21.6$ & 22.6 & $\geq 20.6$ & $\geq 19.9$ & $\geq 18.9$ & & \\
\hline Cha $\mathrm{H} \alpha 9$ & 23.1 & 20.1 & 20.05 & 17.34 & $\mathrm{n} / \mathrm{o}$ & 13.92 & 12.59 & 11.82 & 20.02 & 16 \\
\hline Cha $\mathrm{H} \alpha 10$ & 21.6 & 19.4 & 19.35 & 16.90 & 16.79 & 14.41 & 13.68 & 13.30 & 19.21 & 9 \\
\hline Н $\alpha 10 /$ cc 1 & $\geq 23.1$ & $\geq 23.1$ & 23.3 & 21.7 & 21.8 & 19.0 & 17.6 & 17.1 & & \\
\hline H $\alpha 10 /$ cc 2 & $\geq 23.5$ & $\geq 23.0$ & 24.9 & $\geq 21.2$ & 22.9 & $\geq 18.5$ & $\geq 20.3$ & $\geq 19.9$ & & \\
\hline $\mathrm{H} \alpha 10 / \mathrm{cc} 3$ & $\geq 23.3$ & 23.0 & 23.0 & 21.4 & 21.5 & 19.6 & 18.5 & 17.9 & & \\
\hline Cha $\mathrm{H} \alpha 11$ & 21.9 & 19.9 & 19.69 & 17.35 & 17.41 & 14.72 & 14.03 & 13.60 & 18.95 & 23 \\
\hline $\mathrm{H} \alpha 11 / \mathrm{cc} 1$ & $\geq 22.9$ & $\geq 22.9$ & 23.4 & 22.2 & 22.2 & 21.1 & 19.3 & 18.7 & & \\
\hline Cha $\mathrm{H} \alpha 12$ & 20.6 & 18.3 & 18.34 & 15.58 & 15.72 & $\mathrm{n} / \mathrm{o}$ & $\mathrm{n} / \mathrm{o}$ & $\mathrm{n} / \mathrm{o}$ & 18.03 & 20 \\
\hline
\end{tabular}

Table 4. Observations log of ground-based images.

\begin{tabular}{llrrcc}
\hline Instr. & Cha $\mathrm{H} \alpha$ & filter & exp. & \multicolumn{1}{c}{ date } & $F W H M$ \\
\hline FORS1 & $4,5,8,10,11$ & $V$ & 60s & 26 Jan. 1999 & $0.78^{\prime \prime}$ \\
FORS1 & $4,5,8,10,11$ & $R$ & 60s & 26 Jan. 1999 & $0.87^{\prime \prime}$ \\
FORS1 & $4,5,8,10,11$ & $I$ & 60s & 26 Jan. 1999 & $0.80^{\prime \prime}$ \\
\hline SofI & $4,5,8,10,11$ & $H$ & 200s & 14 Mar. 2000 & $0.68^{\prime \prime}$ \\
SofI & $4,5,8,10,11$ & $K_{\mathrm{s}}$ & 200s & 14 Mar. 2000 & $0.58^{\prime \prime}$ \\
\hline SofI & $4,10,11$ & $J$ & $600 \mathrm{~s}$ & 3 Mar. 2001 & $0.60^{\prime \prime}$ \\
SofI & $4,10,11$ & $H$ & $1200 \mathrm{~s}$ & 3 Mar. 2001 & $0.58^{\prime \prime}$ \\
SofI & $4,10,11$ & $K_{\mathrm{s}}$ & $5400 \mathrm{~s}$ & 3 Mar. 2001 & $0.73^{\prime \prime}$ \\
\hline SofI & 5 & $J$ & $600 \mathrm{~s}$ & 7 Mar. 2001 & $0.74^{\prime \prime}$ \\
SofI & 5 & $K_{\mathrm{s}}$ & $5400 \mathrm{~s}$ & 4 Mar. 2001 & $0.58^{\prime \prime}$ \\
\hline SofI & 8 & $J$ & $600 \mathrm{~s}$ & 3 Mar. 2001 & $0.64^{\prime \prime}$ \\
SofI & 8 & $H$ & $1200 \mathrm{~s}$ & 3 Mar. 2001 & $0.72^{\prime \prime}$ \\
\hline
\end{tabular}

in the ground-based images, we do have already several epochs. However, in the ground-based images, we cannot measure the separation between the primaries and their faint companion candidates as precise as in the HST images (only to \pm 0.5 pixels in the ground-based images), so that we cannot yet measure their proper motions. We can only say that the ground-based separations are not inconsistent with those measured in the HST images.

The typical proper motion of other Cha I T Tauri stars is $\mu_{\alpha} \simeq-29 \mathrm{mas} / \mathrm{yr}$ and $\mu_{\delta} \simeq 11 \mathrm{mas} / \mathrm{yr}$ (Frink et al. 1998; Teixeira et al. 2000). The proper motions of Cha $\mathrm{H} \alpha 1$ to 12 are not known, yet, but we can assume that they are similar to other members, because several other indicators show that Cha $\mathrm{H} \alpha$ 1-12 are members; e.g. they share the typical radial velocity with other Cha I members (Joergens \& Guenther 2001). Given the $R$ - \& I-band HST PC images, we can measure the separation between primary and companion candidate to \pm 33 mas (see below). Hence, with the HST PC pixel scale (45.5 mas), we can check for common proper motion after a few years.

We compiled the $R$ \& I magnitudes of all objects detected in the seven HST images. From the number of objects brighter than a particular companion candidate (Fig. 6), we can estimate the background probability. 


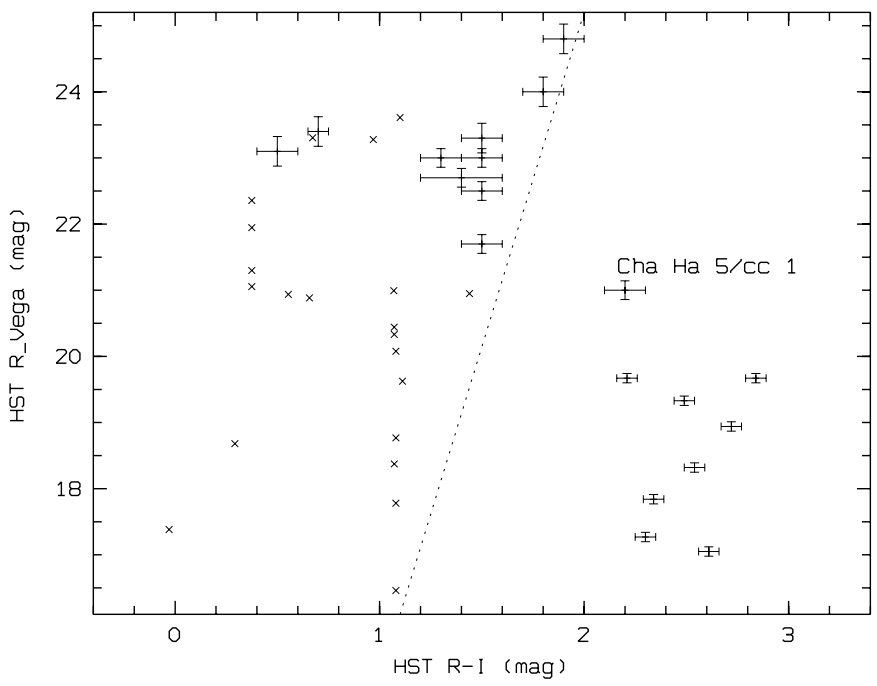

Fig. 4. Optical color-magnitude diagram with $R_{V}$ versus $R_{V}-$ $I_{V}$. We plot all primary objects (with small error bars), companion candidates within $7^{\prime \prime}$ (with larger error bars), and other objects (without error bars) detected in the HST images. All data from Table 3. Note the bimodal distribution: All primaries are located in the lower right of the figure, together with one companion candidate (Cha H $\alpha$ /cc 1), while all other objects are towards the left. Possibly, all objects left of the dotted line are background, while those in the lower right are members of Cha I. Because Cha $\mathrm{H} \alpha 5 / \mathrm{cc} 1$ is located near the primary objects (and because of its small separation), it is the most promising companion candidate.

Lets discuss as example the close companion candidate near Cha $\mathrm{H} \alpha$ 5: There are in total 0.32 (0.23) objects per square arc minute detected in $R$ (and $I$, respectively) with magnitude brighter than Cha $\mathrm{H} \alpha 5 / \mathrm{cc} 1(R=21.2$ and $I=19.2)$, see Fig. 6 for the $\log N-\log S$ of all detected HST sources (primaries, companion candidates, and other certainly unrelated objects). Hence, the probability to find one such faint object within $1.5^{\prime \prime}$ around at least one target in an ensemble of eleven Cha $\mathrm{H} \alpha$ primaries observed is $0.69 \%$ in $R$ (and $0.49 \%$ in $I$ ), i.e. quite small. See also Fig. 8, where it is shown that no other faint objects are detected in the whole PC field-of-view around Cha $\mathrm{H} \alpha 5$.

In the same way, we estimated the background probabilities for all other companion candidates listed in Table 3. They range from the values mentioned above $(\leq 1 \%)$ for the brightest companion candidate (Cha $\mathrm{H} \alpha$ $5 /$ cc 1 at $1.5^{\prime \prime}$ separation) up to $\sim 18 \%$ (at $7^{\prime \prime}$ separation) for the faintest detected companion candidates, Cha $\mathrm{H} \alpha$ 10/cc 2 and Cha $\mathrm{H} \alpha$ 8/cc 2. Given the whole ensemble with eleven primaries observed with HST and 16 detected companion candidates, we can expect a few real companions.

Following the methodology of Lada et al. (1994) we computed the extiction map of the target field making use of the $H$ and $K_{\mathrm{s}}$ band images taken with SofI. The known foreground stars and embedded cloud members (see C1999 and C2000) were not used in computing this map. Because there were no observations of the unreddened background stellar field (necessary for the determination of the zero point of the extinction scale), we adopted a

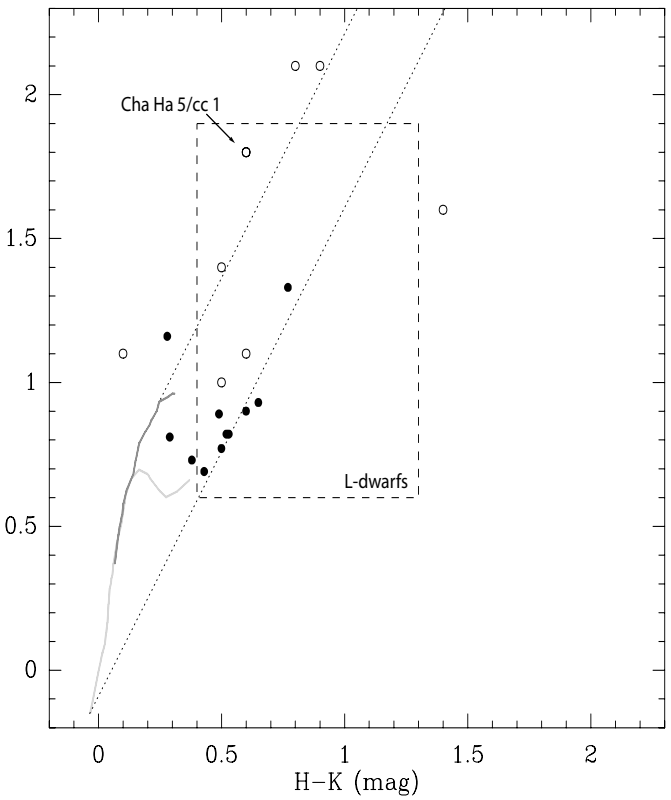

Fig. 5. IR color-color magnitude diagram of primaries (filled circles) and companion candidates (open circles): $J-H$ versus $H-K_{\mathrm{s}}$ with data from Table 3 . Error bars are omitted for clarity, errors range from $\sim 0.07 \mathrm{mag}$ for the brightest primaries to $\sim 0.15 \mathrm{mag}$ for the faintest companion candidates. Solid lines indicate the loci of points corresponding to unreddened main-sequence and giant stars (Bessel \& Brett 1988). The two parallel dotted lines define the reddening band for both main-sequence and giant stars taken from Rieke \& Lebofsky (1985). The dashed box indicates the locii of L-dwarfs according to Burgasser et al. (2001) and Leggett et al. (2002). The companion candidate located to the right of both dotted lines is Cha $\mathrm{H} \alpha 8 / \mathrm{cc} 7$. The lefternmost companion candidate (bluest in $H-K_{\mathrm{s}}$ ) is Cha $\mathrm{H} \alpha 4 /$ cc 2, located near the early T-dwarf area. The most promising companion candidate (Cha $\mathrm{H} \alpha 5 / \mathrm{cc} 1$ ) is indicated by an arrow.

$<H-K_{\mathrm{s}}>$ color for this background. Regarding this choice, Alves et al. (1998) found $\left\langle H-K_{\mathrm{S}}\right\rangle=0.20 \pm$ 0.13 mag towards a complicated region in Cygnus, essentially at the Galactic plane, while Alves et al. (2001) found $\left.<H-K_{\mathrm{s}}\right\rangle=0.12 \pm 0.08 \mathrm{mag}$ towards a clean lineof-sight towards the Galactic bulge, at $b \simeq 7^{\circ}$. The stellar background towards the Chamaeleon complex is probably even better behaved, because it lies at $b \simeq 15^{\circ}$. On the other hand, the $<H-K_{\mathrm{s}}>$ color of the North Galactic pole from 2MASS data is also $\sim 0.12$ mag (M. Lombardi, private communication), so we decided to adopt this as the mean Chamaeleon background color. We also adopt a conservative intrinsic dispersion in this measurement of $0.13 \mathrm{mag}$, i.e. an intrinsic extinction measurement $\mathrm{rms}$ of $A_{\mathrm{V}} \simeq 2 \mathrm{mag}$.

We show our map in Fig. 7 for the area around Cha $\mathrm{H} \alpha 4,5,8,10$, and 11 , where companion candidates were detected. The spatial resolution of the map is $50^{\prime \prime}$ and the extinction $A_{V}$ ranges from 4 to $22 \mathrm{mag}$. Each position of this map is associated with a total extinction which includes the whole Cha I dark cloud and its foreand background. 


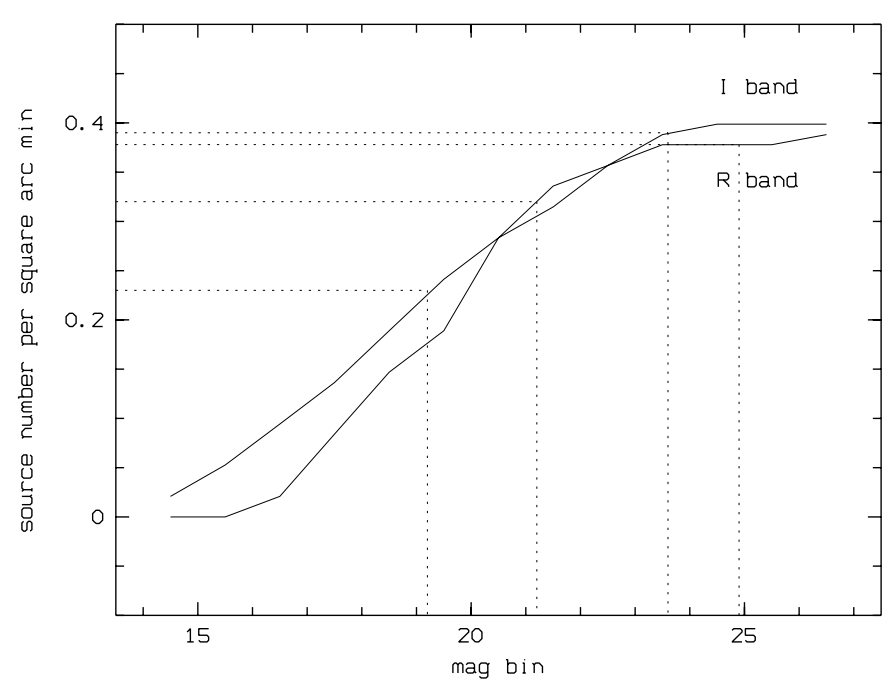

Fig. 6. Number of sources brighter than limiting magnitude per square arc minute versus magnitude for $R$ - and $I$-band HST images. We used these $\log N-\log S$ curves for estimating the probability that a certain companion candidate actually is an unrelated background object behind the Cha I dark cloud. The broken lines show those data for the brightest (Cha H $\alpha$ / cc 1) and faintest (Cha H $\alpha 10 /$ cc 2 in $R$ and 8/cc 2 in $I$ ) companion candidates.

C2000 derived the individual reddening by comparing the observed colors to intrinsic colors for the known spectral types. Those absorptions range from $A_{V} \simeq 0.0$ to $1.0 \mathrm{mag}(\mathrm{C} 2000)$ and are smaller than the extinction values towards their immediate background, as measured in the extinction map. The total extinction towards the immediate background of the primaries shown in Fig. 7 range from $A_{V} \simeq 6$ to $8 \mathrm{mag}$. Hence, the primaries are most certainly located near the front edge of the dark cloud.

\section{The promising candidate near $\mathrm{Cha} \mathrm{H}_{\alpha} 5$}

Cha $\mathrm{H} \alpha 5$ is an M6 dwarf with $\mathrm{H} \alpha$ and X-ray emission as well as Lithium absorption; no radial velocity variations were detected in UVES spectra, consistent with membership to the Cha I association (C1999, C2000; Joergens $\&$ Guenther 2001). Based on a comparison of its location in the $H-R$ diagram with different tracks and isochrones, Cha $\mathrm{H} \alpha 5$ has a mass of $\sim 0.10 M_{\odot}$ and an age of $\sim 2$ Myrs, i.e. co-eval with the other M6- to M8type objects Cha H $\alpha 1$ to 12 (C2000). Hence, it is either a very low-mass $\mathrm{T}$ Tauri star or a relatively massive brown dwarf.

A faint companion candidate is located $1.085 \pm 0.027^{\prime \prime} \mathrm{S}$ and $1.036 \pm 0.028^{\prime \prime} \mathrm{W}$ of $\mathrm{Cha} \mathrm{H} \alpha 5$, i.e. at a separation of $1.500 \pm 0.033^{\prime \prime}$ with a position angle of $223.66 \pm 0.10^{\circ}$ (taking into account an uncertainty of $\pm 0.08^{\circ}$ in the N-S alignment of the detector), identical in both the $R$ - and $I$-band image. The companion candidate is not detected in $\mathrm{H} \alpha$, while all primary objects including Cha $\mathrm{H} \alpha 5$ are detected. The HST $R$ - and $I$-band images of Cha $\mathrm{H} \alpha 5$ with its faint companion candidate are shown in Fig. 8.

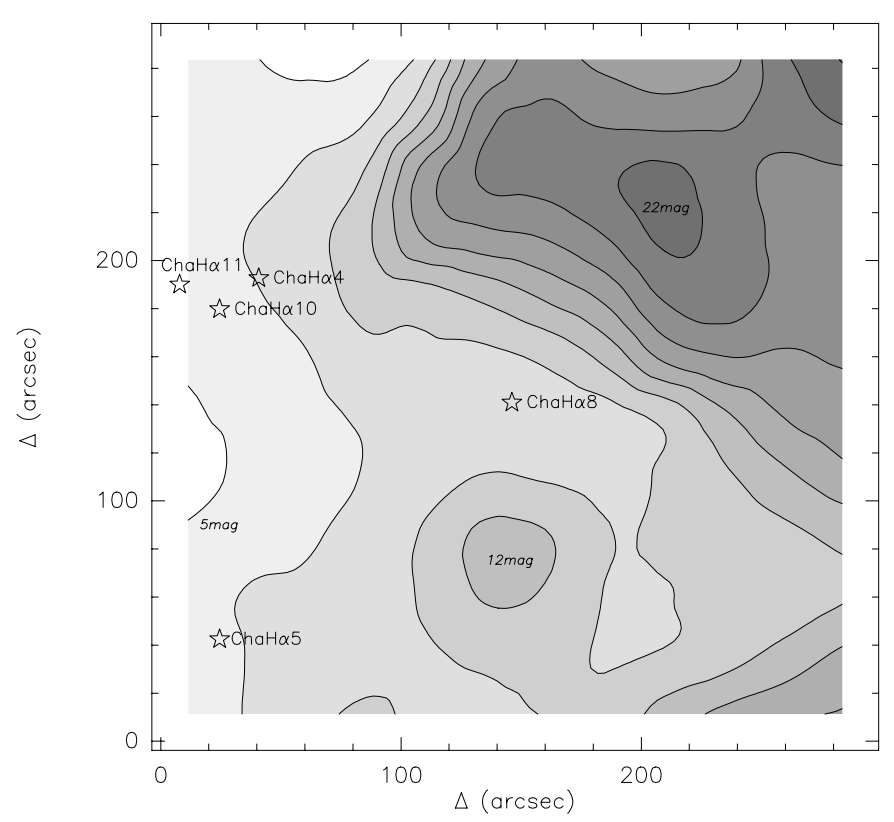

Fig. 7. Background map: Dust extinction map of the studied region as derived from the IR $(H-K)$ observations of background sources. Contours start at 5 visual mag of extinction and increase in steps of 2 mag up to 21 mag of visual extinction. The effective resolution of the map is $50^{\prime \prime}(\sim 0.04 \mathrm{pc}$ at the distance of Cha I). The positions of Cha $\mathrm{H} \alpha 4,5,8,10$, and 11 , where companion candidates were detected, are also indicated.

The companion candidate is also marginally resolved in the ground-based $V R I J H K_{\mathrm{s}}$ images (Figs. 9 and 10). In the ground-based image, where it is resolved best, the SofI $K_{\mathrm{s}}$-band image from March 2001, the separation measured is $1.38 \pm 0.21^{\prime \prime}$ at a position angle of $220.6 \pm 8.7^{\circ}$, i.e. much less precise than the HST data, but not inconsistent with low differential proper motion.

In the $H$ - and $K_{\mathrm{s}}$-band images (Fig. 10), we could obtain the magnitude of the companion candidate by normal aperture photometry. However, in the VRIJ images, this was not possible, because of the even higher dynamic range.

In the $V$ - and $J$-band images, we first obtained the flux in each pixel within $4^{\prime \prime}$ of the center of the primary, then plotted the flux as a function of the separation to the primary (Fig. 9, right panels). Those functions are a superposition of two Moffat functions (Moffat 1969), one for the bright primary and one for the faint companion candidate located in the wing of the primary's PSF, both with the same $F W H M$. The faint companion candidate is detected as a small bump in the primary's PSF. Then, we derived the peak flux of the companion candidate at $1.5^{\prime \prime}$ separation from the primary by subtracting the flux value of the upper envelope by the flux value of the lower envelope, both at $1.5^{\prime \prime}$. From this flux and the peak flux of the primary, we derived the magnitude difference between primary and companion candidate. The same procedure was performed for the VLT $R$ - and $I$-band images, where the companion candidate is also detected in the PSF wing. The magnitudes derived from these VLT $R$ - and $I$-band 

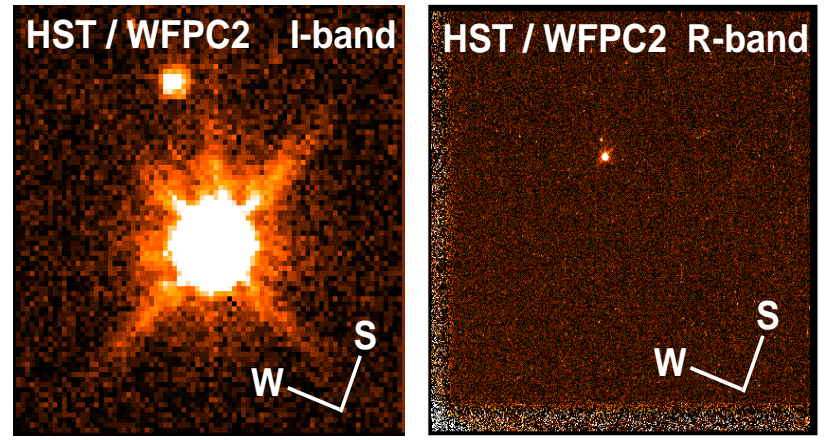

Fig. 8. HST R- \& I-band images of Cha $\mathrm{H} \alpha 5$ and surrounding field. Left: $I$-band image $\left(4^{\prime \prime} \times 4^{\prime \prime}\right.$ field $)$. A companion candidate $1.5^{\prime \prime} \mathrm{SW}$ of Cha $\mathrm{H} \alpha 5$ is detected. Right: $R$-band image with the whole PC field $\left(\sim 15^{\prime \prime} \times 15^{\prime \prime}\right)$, where no objects other than the primary and one companion candidate are detected. Also in $\mathrm{I}$ and $\mathrm{H} \alpha$, no other objects are detected.

images are consistent with the HST data, where the companion candidate is well detected and well resolved from the primary $( \pm 0.10 \mathrm{mag}$ in $R$ and $\pm 0.19 \mathrm{mag}$ in $I)$. Hence, we can trust our procedure.

For the line-of-sight absorption in the direction of Cha $\mathrm{H} \alpha 5$, we measure $A_{V} \simeq 6.5 \mathrm{mag}$ (see Fig. 7), i.e. several times more than foreground to $\mathrm{Cha} \mathrm{H} \alpha 5$ $\left(A_{V}=0.98 \mathrm{mag}, \mathrm{C} 2000\right)$. Apart from the fact that this object could be a true companion, we have to consider several alternatives:

- The $V H K_{\mathrm{s}}$ colors of the companion candidate are consistent with a $\mathrm{K}$ giant reddened by $A_{\mathrm{V}} \simeq 6.5 \mathrm{mag}$, which would be located at $\sim 11 \mathrm{kpc}$, i.e. $\sim 3 \mathrm{kpc}$ from the galactic plane, but its dereddened $J-H$ color is $1.5 \sigma$ redder than a $\mathrm{K}$ giant.

- The unreddened optical colors of the companion candidate are consistent with those of an M 5-6 dwarf, which would lie foreground to the extinction. However, the $H \& K_{\mathrm{s}}$ flux would be too bright and the $V-H$ and $V-K_{\mathrm{S}}$ colors would be $4 \sigma$ redder than a M 5-6 dwarf. The bright $H$ and $K_{\mathrm{s}}$ fluxes could be IR excess due to circumstellar material, but such strong excess $\left(\sim 1.5 \mathrm{mag}\right.$ in $H$ and $\left.K_{\mathrm{s}}\right)$ was never observed for a mid-M type star.

- If the object would be a moderately extincted $\left(A_{V} \simeq\right.$ $1 \mathrm{mag}$ ) late M-dwarf in the background, its optical colors would be $2 \sigma$ too blue for a late M-dwarf.

Hence, the companion candidate is unlikely to be a background giant or a foreground dwarf. As discussed in the previous section, a background object is very unlikely anyway. Even considering the whole ensemble observed, the probability to find one such object within $1.5^{\prime \prime}$ of one our targets is $\leq 0.7 \%$. The probability for the object being a foreground dwarf will be considered below.

For a companion to $\mathrm{Cha} \mathrm{H} \alpha 5$, we can assume the same extinction as towards the primary $\left(A_{I}=0.47 \mathrm{mag}\right.$, C2000). The error should be $\sim 0.07 \mathrm{mag}$, as estimated from the errors in $R$ and $I$. With the Rieke \& Lebofsky (1985) extinction law, we can then estimate dereddened colors
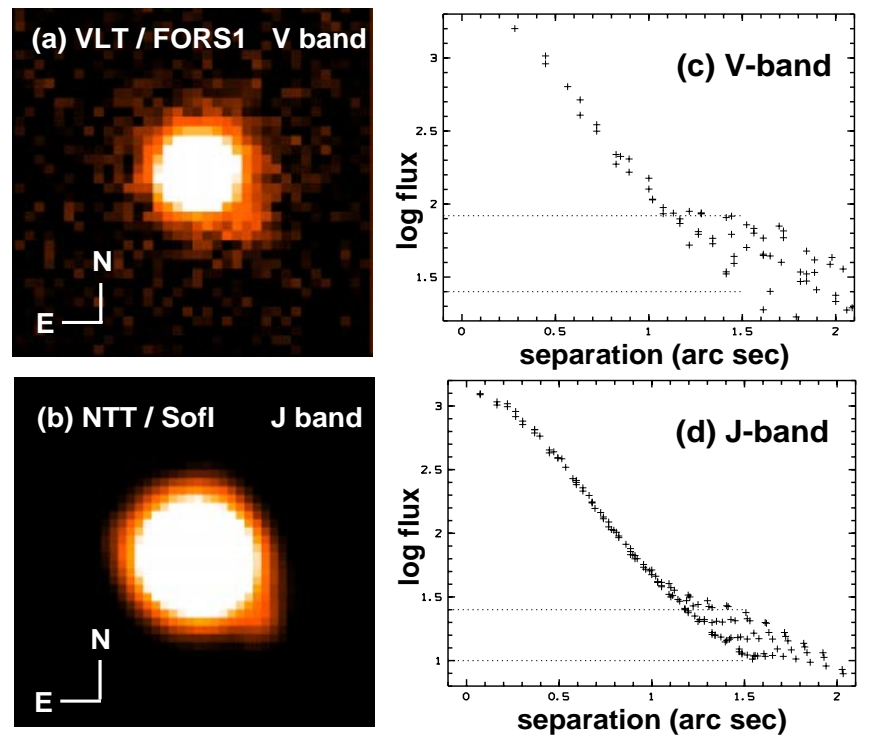

Fig. 9. $V$ - \& $J$-band images of $\mathrm{Cha} \mathrm{H} \alpha 5$ and its companion candidate $1.5^{\prime \prime}$ to the SW. a) VLT V-band image. b) NTT $J$-band image. c) and d) Cha $\mathrm{H} \alpha 5$ PSF with the faint companion candidate as bump in the primary's wing, plotted as log of flux versus separation in arc sec from the primary, in $V$ and $J$, respectively. We computed the companion's magnitude by subtracting the contribution of the primary at $1.5^{\prime \prime}$ separation (lower envelope of the plotted points) from the peak of the bump (upper envelope) and comparing it to the observed flux and known magnitude of the primary. The upper horizontal dotted lines show the maximum flux of the companion in $V$ and $J$, respectively, and the lower dotted lines show the flux contribution of the primary at $1.5^{\prime \prime}$ separation in $V$ and $J$, respectively.
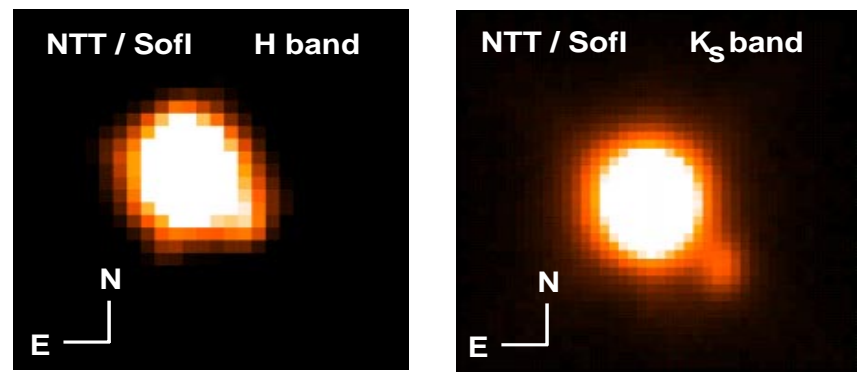

Fig. 10. NTT $H$ - \& $K_{\mathrm{s}}$-band images of Cha $\mathrm{H} \alpha 5$.

(Table 5), to be compared with intrinsic colors of lateM and L dwarfs (see e.g. Kirkpatrick et al. 1999, 2000, henceforth K1999 and K2000). Few $V$-band magnitudes of L-dwarfs are available so far, so that we could not compare our object to typical L-dwarfs in this regard. The comparison of $R I J H K_{\mathrm{s}}$ colors in Table 5 shows that a spectral type of early- to mid-L is most likely for our object. The observed magnitude difference between the M6 primary and its companion of 3.8 to $4.7 \mathrm{mag}$ and also its non-detection in $\mathrm{H} \alpha$ are consistent with spectral type $\mathrm{L}$ (K1999, K2000). As seen in Table 5, neither all the observed nor all the dereddened colors are perfectly consistent with any single spectral type sub-class, but this is not surprising, because all (or most) known L-dwarfs are 
Table 5. Colors of Cha $\mathrm{H} \alpha 5 / \mathrm{cc} 1$ and typical L dwarfs.

\begin{tabular}{lcccll}
\hline & $\begin{array}{c}\text { dereddened } \\
\text { color }[\mathrm{mag}]\end{array}$ & $\begin{array}{c}\text { mean } \\
\text { L0 }\end{array}$ & $\begin{array}{c}\text { best } \\
\text { guess }\end{array}$ & $\begin{array}{l}\text { possible } \\
\text { range }\end{array}$ & ref. \\
\hline$(R-I)_{0}$ & $1.46 \pm 0.25$ & 2.0 & M7 & M6-L2 & 5 \\
$\left(R-K_{\mathrm{s}}\right)_{0}$ & $5.93 \pm 0.33$ & 6.0 & M8 & M6-L2 & 5 \\
$(I-J)_{0}$ & $2.21 \pm 0.28$ & 2.5 & M8 & M8-L4 & 1,3 \\
$(I-H)_{0}$ & $3.90 \pm 0.28$ & 3.5 & L1 & M9.5-L1.5 & 5 \\
$\left(I-K_{\mathrm{s}}\right)_{0}$ & $4.47 \pm 0.34$ & 4.2 & L0 & M9-L4 & 1,5 \\
$(J-H)_{0}$ & $1.69 \pm 0.29$ & 1.0 & L5 & L4-L8 & $2-4$ \\
$\left(J-K_{\mathrm{s}}\right)_{0}$ & $2.26 \pm 0.34$ & 1.4 & L5 & L2-L8 & $1-4$ \\
$\left(H-K_{\mathrm{s}}\right)_{0}$ & $0.56 \pm 0.35$ & 0.6 & L1 & L0-L5.5 & $2,4,5$ \\
\hline
\end{tabular}

Ref.: (1) K1999, (2) K2000 (3) Leggett et al. (2002), (4)

Burgasser et al. (2001), (5) astro.nmsu.edu/ ${ }^{\sim}$ crom/bdwarfs .

much older and more massive, and evolutionary effects in the atmosphere are expected. The $R I J K_{\mathrm{s}}$ colors of our companion candidate are consistent with those of young early-L dwarfs in $\sigma$ Ori (Bejar et al. 1999).

The Cha $\mathrm{H} \alpha 5$ companion candidate could also be an isolated young L dwarf in the Cha I association, rather than a companion of Cha $\mathrm{H} \alpha 5$. This is, however, very unlikely: Even if there are 10 times more L-dwarfs in Cha I than M-dwarfs (there are 19 M-dwarfs known in Cha I), then we would expect to find in total $0.01 \mathrm{~L}$-dwarfs within $1.5^{\prime \prime}$ around the eleven Cha $\mathrm{H} \alpha$ objects observed.

There is an L2.5 dwarf with similar color ${ }^{7}$, so our object could be an old foreground L2.5 dwarf. Reid et al. (1999) expect $4634 \mathrm{~L}$-dwarfs with $K_{\mathrm{s}} \leq 14.5 \mathrm{mag}$ on the whole sky, so the probability to find one L-dwarf within $1.5^{\prime \prime}$ of the eleven Cha $\mathrm{H} \alpha$ objects observed is $0.007 \%$.

With an estimated spectral type of $\mathrm{L}$, the temperature is $\sim 2200$ to $1400 \mathrm{~K}$ (K1999, K2000, Basri et al. 2000; Burgasser et al. 2001). For the age, we assume 1 to 5 Myrs, as the age of Cha $\mathrm{H} \alpha 5$ according to Burrows et al. (1997) and Baraffe et al. (1998) models, see C2000.

If we use $B . C \cdot \mathrm{I}=0.59 \mathrm{mag}$ as given by $\mathrm{C} 2000$ for M8 also for our companion candidate, then we obtain $\log \left(L_{\mathrm{bol}} / L_{\odot}\right)=-3.0$. With B.C.K $=3.2 \mathrm{mag}$ as given by Leggett et al. (2002) for L1, we obtain $\log \left(L_{\mathrm{bol}} / L_{\odot}\right)=$ -2.7 . Considering the magnitude difference in $V R I J H K_{\mathrm{s}}$ between primary and companion being 3.8 to 4.7 mag and the primary having $\log \left(L_{\mathrm{bol}} / L_{\odot}\right)=-1.31(\mathrm{C} 2000)$, the companion has $\log \left(L_{\text {bol }} / L_{\odot}\right)=-2.8$ to -3.2 . Hence, we will use $\log \left(L_{\mathrm{bol}} / L_{\odot}\right)=-3.0 \pm 0.3$.

This luminosity, $T_{\text {eff }} \simeq 1400$ to $2200 \mathrm{~K}$, and an age of 1 to 5 Myrs is consistent with a $\sim 3$ to $15 M_{\text {jup }}$ mass object according to Table 3 and Figs. 7, 9, and 11 in Burrows et al. (1997). According to Figs. 6 and 7 in Chabrier \& Baraffe (2000), an object with the given $L_{\text {bol }}, T_{\text {eff }}$, and age would have a mass of $\sim 7$ to $15 M_{\text {jup }}$. Hence, the faint object could be a planet or a brown dwarf, if really a companion of Cha $\mathrm{H} \alpha 5$. However, all these models may be uncertain at this very young ages, because they start after the formation with assumed thermal structures. Grey

\footnotetext{
7 2MASS091838.2+213406 with $I \simeq 18.68, K_{\mathrm{s}}=4.21 \pm 0.07$, and $H-K_{\mathrm{s}}=0.43 \pm 0.09 \mathrm{mag}$ at $\sim 45 \mathrm{pc}(\mathrm{K} 1999)$
}

dynamical calculations of the formation process locate this candidate between the tracks of a young $10 M_{\text {jup }}$ and a $1 M_{\text {sat }}$ protoplanet (G. Wuchterl, pers. comm.).

The separation between Cha $\mathrm{H} \alpha 5$ and its possible companion is $1.5^{\prime \prime}$ corresponding to a projected physical separation of $210 \mathrm{AU}$ (at $140 \mathrm{pc}$ ), i.e. larger than expected for planets, but not larger than typical circumstellar disks. Such a large separation should not be excluded, not even for planetary companions, because of possible dycamical interaction between proto-planets (by which one gets kicked out) and also, because some of the radial velocity planet candidates have large eccentricities, i.e. are located at large separations most of the time.

Confirmation of this candidate as true companion can be done best and soon by spectroscopy, which is scheduled for the VLT for early 2002. If confirmed as true companion, this object may be the lowest-mass companion ever detected by direct imaging.

\section{Is Cha $\mathrm{H} \alpha 2$ a close binary?}

In the previous sections, we investigated wide companions, which were clearly resolved, at least in the HST images. To search for very close companions, we determined possible elongations of the point-spread-functions (PSF) of the primaries, measured as ratio between the Full Width at Half-Maximum $(F W H M)$ in the direction of the strongest elongation and the $F W H M$ in the direction perpendicular to the elongation. In Fig. 11, we plot the observed PSF elongation versus the magnitude for all measurements in $R, I$, and $\mathrm{H} \alpha$.

While most PSFs are consistent with a single point source (elongation $\sim 1$ ), there is one notable outlyer, namely Cha $\mathrm{H} \alpha 2$, where the PSF for both the $R$ - and $\mathrm{H} \alpha$ observation is elongated by a factor of $\sim 1.5$ towards the NE-SW direction (not observed in $I$ due to technical problems with WFPC2 shutter). All three other sources detected on chip WF4 (like Cha $\mathrm{H} \alpha 2$ ) as well as Cha $\mathrm{H} \alpha 9$ (on WF2 chip in the same observation) are consistent with point sources. The Cha $\mathrm{H} \alpha 2 \mathrm{PSF}$ are $\sim 5.5 \sigma$ deviant from the other Cha $\mathrm{H} \alpha$ primaries. Hence, we do have an indication for Cha $\mathrm{H} \alpha 2$ being a close binary.

Cha $\mathrm{H} \alpha 2$ is elongated in the same direction in both $R$ and $\mathrm{H} \alpha$. Investigating the $R$-band image of Cha $\mathrm{H} \alpha 2$ in more detail shows that the SW component is a factor of $\sim 1.56$ brighter than the NE component, where the flux of the fainter component still includes the flux of the brighter one at that separation. After subtracting the PSF of the brighter (SW) component, the NE component is fainter by $\sim 0.2 \mathrm{mag}$. The position angle of the NE companion candidate relative to the SW primary is $\sim 40^{\circ}$ (measured as usually from $\mathrm{N}$ to $\mathrm{E}$ ), and the separation between the close pair is almost $0.2^{\prime \prime}$, i.e. slightly less than two pixels. As unresolved object, Cha $\mathrm{H} \alpha 2$ has $R_{V}=17.42 \mathrm{mag}$ (Table 3 ), so that the two components would have $\sim 17.45$ and $\sim 17.65 \mathrm{mag}$ in $R_{V}$. The probability to find an $R_{V} \simeq$ 17.65 mag object with $\sim 0.2^{\prime \prime}$ separation by chance around 


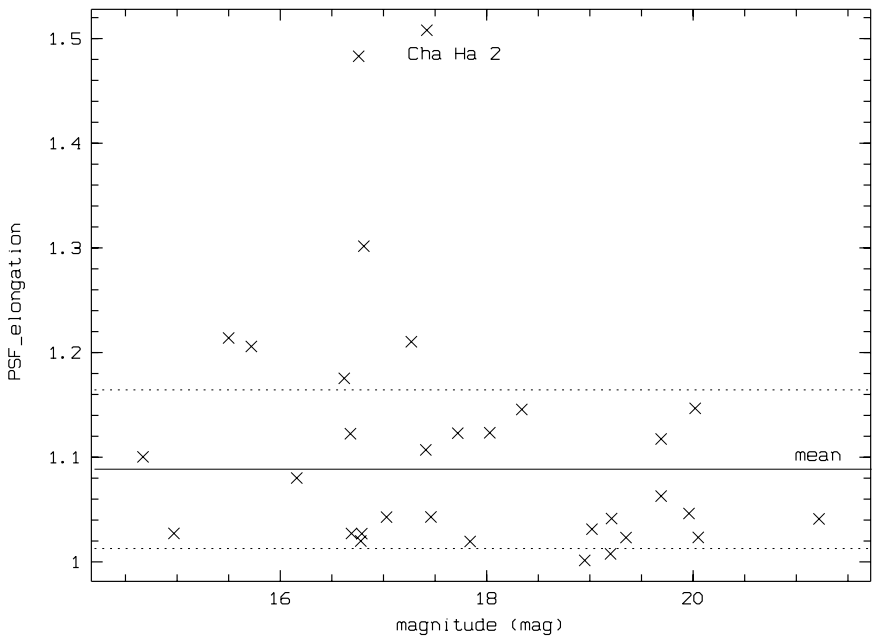

Fig. 11. Apparent elongation of the point-spread-functions (PSF) versus magnitude for the primaries (and the Cha $\mathrm{H} \alpha 5$ companion candidate). Only Cha $\mathrm{H} \alpha 2$ (observed in $R$ and $\mathrm{H} \alpha$, not in $I$ ) appears to be elongated. All other PSFs have a mean elongation of $1.089 \pm 0.076$ plotted as full line (with broken lines indicating its $1 \sigma$ standard deviation range). The Cha $\mathrm{H} \alpha 2$ PSFs with 1.51 in $R$ (and 1.48 in $\mathrm{H} \alpha$ ), that is $\sim 5.5 \sigma$ deviant from the mean, hence possibly indicating a very close companion.

11 primaries observed is only $3.6 \times 10^{-3} \%$ (obtained from Fig. 6).

Even though the PSF elongation of Cha $\mathrm{H} \alpha 2$ is cleary larger than in all other objects, we regard it as candidate binary. See Fig. 12 for the HST image of this object. We cannot completely exclude that the apparent elongation could be due to, e.g., bad pixels. Further obvervations are needed to confirm the apparent elongation. The groundbased FORS1 and SofI images do not have sufficient resolution to detect this elongation.

Cha $\mathrm{H} \alpha 2$ is located close to the sub-stellar limit, i.e. either a very low-mass star or a relatively massive brown dwarf, according to both Burrows et al. (1997) and Baraffe et al. (1998) tracks, see C2000. As approximately equal-brightness and, hence equal-mass binary, both components would still be very close to the sub-stellar limit, i.e. either low-mass stars or brown dwarfs. More precise imaging photometry (e.g. with the PC or with NICMOS) and resolved spectroscopy may resolve this issue. A separation of only $\sim 0.2^{\prime \prime}$ would correspond to only $\sim 28 \mathrm{AU}$ at $140 \mathrm{pc}$, so that one could hope to see orbital motion within a few decades.

\section{Discussion}

From the images of Cha $\mathrm{H} \alpha 5$ and 8 and their companion candidates observed on the PC chip, we determined the dynamic range achieved on the PC chip. From the deepest exposures with Cha $\mathrm{H} \alpha 4,10, \& 11$ on WF4 and Cha $\mathrm{H} \alpha 8$ on WF2, we determined the dynamic range achieved on the WF chips. In Fig. 13, the dynamic range is plotted as flux ratio versus separation, with flux ratio being the
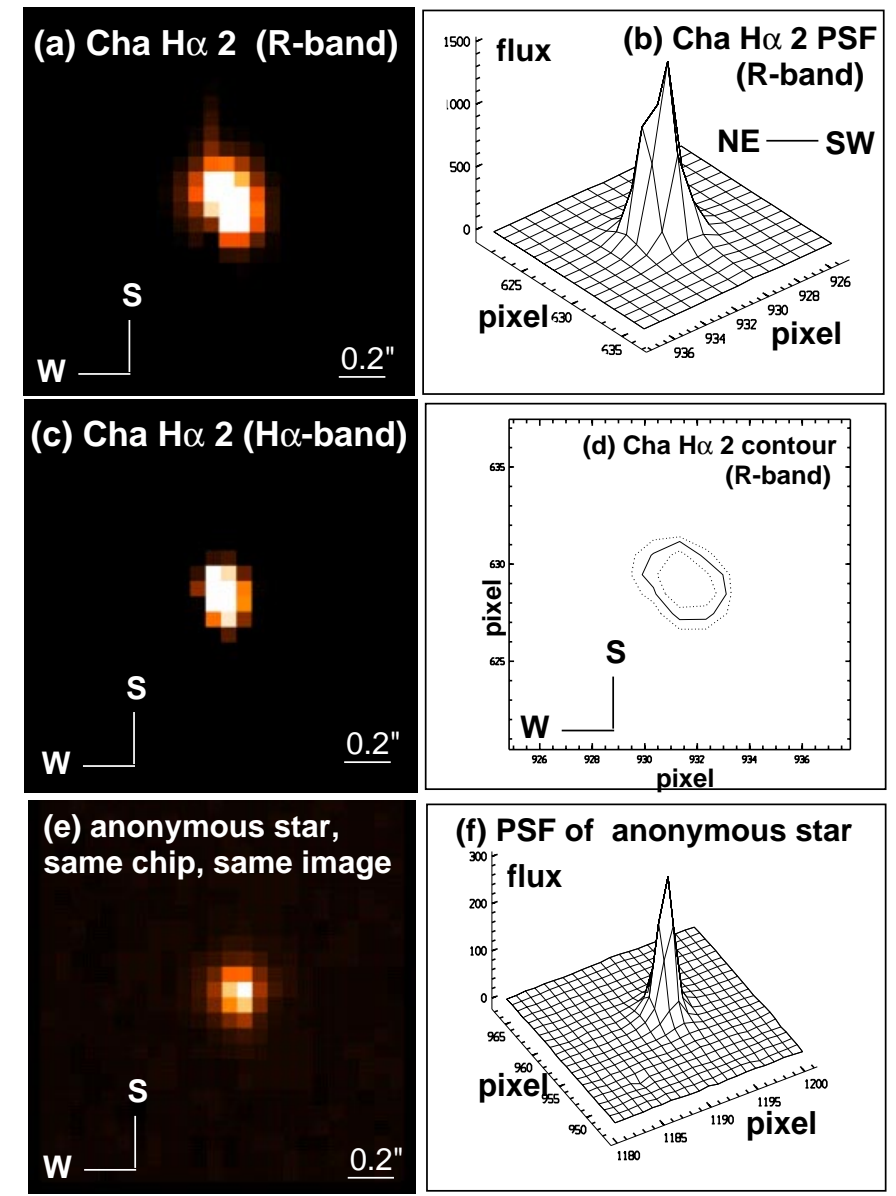

Fig. 12. The possible marginal $\sim 0.2^{\prime \prime}$ elongation of Cha $\mathrm{H} \alpha 2$ : a) HST WF4 chip $R$-band image of Cha $\mathrm{H} \alpha 2$. b) 3D representation of the PSF of Cha $\mathrm{H} \alpha 2$ ( $R$-band) showing a faint secondary bump to the NE. c) HST WF4 chip image of Cha $\mathrm{H} \alpha 2$ in $\mathrm{H} \alpha$, again slightly elongated. d) $2 \mathrm{D}$ representation of the PSF of Cha $\mathrm{H} \alpha 2$ ( $R$-band) showing the small SW-NE elongation. e) HST WF4 chip $R$-band image of another star (same chip, same observation). f) PSF of that other star. While the $\mathrm{PSF}$ of Cha $\mathrm{H} \alpha 2$ is always slightly elongated, the PSF of the (anonymous) comparison star is consistent with a point source.

ratio between either a companion or the $3 \sigma$ background level and the particular primary ( $I$-band).

From the mean (and faintest, respectively) $I$-band magnitude of the primaries (being $16.0 \mathrm{mag}$ and 17.5, respectively) and the dynamic range limit (log flux ratio being 4, i.e. a magnitude difference of 10 mag outside of $2^{\prime \prime}$ with the $\mathrm{PC}$ ), we can then obtain the magnitude limit for detectable companions, namely 26 mag (27.5 mag), or one mag brighter at $1^{\prime \prime}$ separation (and 2 mag brighter for the WF chips). For an assumed $I-K_{\mathrm{s}}$ color index of $\sim 4.5$ mag for an L- or T- dwarf, this would correspond to a limit of $21.5 \mathrm{mag}(23.0 \mathrm{mag})$ in $K_{\mathrm{s}}$. This limiting magnitude at a distance of $140 \mathrm{pc}$ and an age of 2 Myrs would correspond to a limiting companion mass of $\leq 1 M_{\text {jup }}$ according to table 1 in Burrows et al. (1997) with B.C.K $=2 \mathrm{mag}$ (as for Gl $229 \mathrm{~B}$, Leggett et al. 1999). 


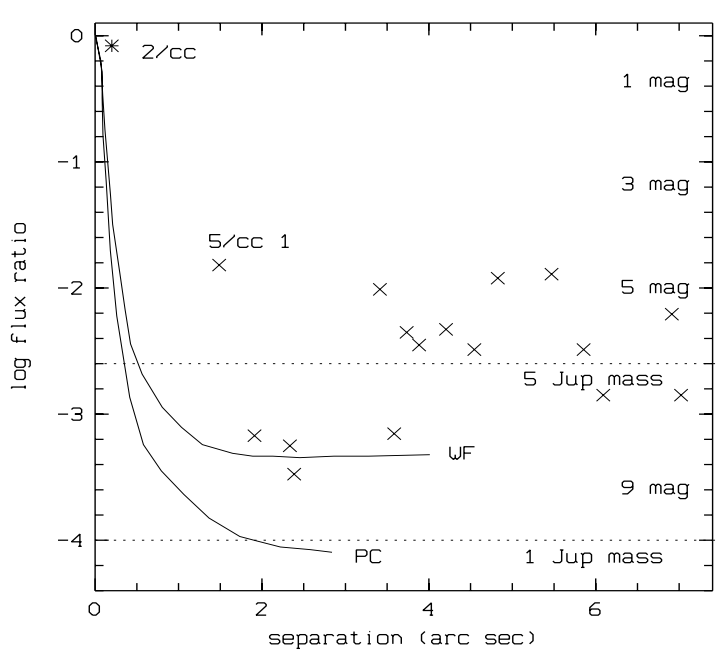

Fig. 13. Dynamic range for our HST WFPC2 images: We plot the log of the flux ratio between primary and detected companion candidates (crosses) with $I$-band magnitudes from Table 3 (magnitude difference is given on the right-hand side $y$-axis). Also shown is the $I$-band dynamic range curve obtained on the $\mathrm{PC}$ and WF chips (determined as flux ratio between primary and flux being $3 \sigma$ above the background level). The two dotted lines show the approximate flux ratio for 5 and $1 M_{\text {jup }}$ mass companions (at 2 Myrs and $140 \mathrm{pc}$ ) around a primary (calculated for the mean primary $I$-band magnitude of $16 \mathrm{mag}$ ) according to Burrows et al. (1997) models. We indicate the locations of the very close binary candidate Cha $\mathrm{H} \alpha 2$ (as star symbol in the upper left) and the most promising resolved companion candidate Cha $\mathrm{H} \alpha 5 / \mathrm{cc} 1$.

Hence, we should have been able to have detected all companions with mass above $\sim 1 M_{\text {jup }}$ outside of $2^{\prime \prime}$ (320 AU) and all companions down to a few $M_{\text {jup }}$ at $\sim 100$ AU. Outside of $0.35^{\prime \prime}$ (50 AU), we should have detected all companions with masses above $\sim 5 M_{\text {jup }}\left(K_{\mathrm{s}} \simeq\right.$ $18 \mathrm{mag})$.

If isolated brown dwarfs are ejected early in the accretion phase (Reipurth \& Clarke 2001) or some time during the pre-main sequence evolution (Sterzik et al. 2001), one should expect more high-mass-ratio binaries among very young stars than among old stars, which we can check with our sample. We observed 11 M6-M8-type primaries with HST and found 16 clearly resolved wide companion candidates around five of them plus one additional very close $\left(0.2^{\prime \prime}\right)$ candidate binary. From the background population, we estimated the probability for each candidate to be a true companion, so that we can expect a few real companions among the candidates. From the optical and IR colors, only two of the candidates could be L- or T-type objects (Cha $\mathrm{H} \alpha 5 / \mathrm{cc} 1$ and $\mathrm{Cha} \mathrm{H} \alpha$ 4/cc 2). Up to two true sub-stellar companions around 11 primaries correspond to a percentage of $18 \pm 13 \%$. All these detected companion candidates (as well as un-detected, but detectable companions) would have separations in the range from $\sim 100 \mathrm{AU}$ (resolution limit) to $1000 \mathrm{AU}$ (somewhat arbitrary upper limit) and high mass-ratios of 10 to 100 (with the ratio being the mass of the primary devided by mass of the companion). If Cha $\mathrm{H} \alpha 2$ is a close binary (Sect. 7), then the secondary could also be a sub-stellar companion, so that we would then have $\leq 3$ sub-stellar companions around 11 primaries, i.e. $27 \pm 16 \%$.

This should be compared to the frequency and orbit characteristics of other high-mass-ratio binaries with large separations, i.e. brown dwarfs in orbit around a star. Recently, Gizis et al. (2001) estimated the frequency of wide, visual, old L- and T-type brown dwarf companions to normal stars to be $18 \pm 14 \%$. This number is consistent with our estimate given above, so that we find no evidence for the ejection scenario. However, because of the large error bars due to small-number-statistics, we do not have sufficient evidence to make a strong statement in this regard. In addition, the surveys discussed by Gizis et al. (2001) and our survey have different dynamical ranges and detection limits.

We also have to refrain from comparing our results with previous surveys for companions around young lowmass stars in Chamaeleon (Reipurth \& Zinnecker 1993; Brandner et al. 1996; Ghez et al. 1997; Köhler 2002), because our sample of primaries (M6- to M8-type bona-fide and candidate brown dwarfs) is different from the previously surveyed samples (G- to early M-type T Tauri stars) and because the previous surveys were restricted to smaller dynamical ranges, up to a magnitude difference of 5 mag between primary and companion candidate, while all but one (Cha $\mathrm{H} \alpha 5)$ or two (Cha $\mathrm{H} \alpha 2$ ) of our companion candidates have larger magnitude differences.

In our observations, we found one promising companion candidate (Cha $\mathrm{H} \alpha 5 / \mathrm{cc} 1$ ), which could be a brown dwarf (or even giant planet) companion. The other candidates are all fainter and often detected only in I. If they were true companions, they would all be giant planets, given their magnitudes, assuming the same age and distance as towards the primaries. However, they are all at physical separations $\geq 200 \mathrm{AU}$, which is not expected for planets, but should not be excluded. Follow-up 2nd epoch imaging and/or spectroscopy will show, whether and which of our companion candidates are truely cool and bound.

Acknowledgements. We would like to thank the support staff at VLT, NTT, and STScI, especially our program coordinator Galina Soutchkova, as well as Günther Wuchterl, Eike Guenther, and Eduardo Martín for useful discussion. $\mathrm{RN}$ and WB acknowledge the hospitality and support from the Institute for Astronomy at the University of Hawai'i, where part of the work was carried out. RN acknowledges financial support from the BMBF through DLR grant 50 OR 0003. VJ acknowledges financial support from the DFG Schwerpunktprogramm Sternentstehung.

\section{References}

Alves, J., Lada, C. J., Lada, E. A., Kenyon, S. J., \& Phelps, R. 1998, ApJ, 506, 292

Alves, J., Lada, C. J., \& Lada, E. A. 2001, Nature, 409, 159 
Baraffe, I., Chabrier, G., Allard, F., \& Hauschildt, P. H. 1998, A\&A, 337, 403

Basri, G., \& Martín, E. L. 1999, ApJ, 118, 2460

Basri, G., Mohanty, S., Allard, F., et al. 2000, ApJ, 538, 363

Bejar, V., Zapatero-Osorio, M., \& Rebolo, R. 1999, ApJ, 521, 671

Bessel, M. S., \& Brett, M. J. 1988, PASP, 100, 1134

Brandner, W., Alcalá, J. M., Kunkel, M., Moneti, A., \& Zinnecker, H. 1996, A\&A, 307, 121

Brandner, W., Zinnecker, H., Alcalá, J. M., et al. 2000, AJ, 120,950

Burgasser, A. J., Kirkpatrick, J. D., Brown, M. E., et al. 2001, preprint [astro-ph/0108452]

Burrows, A., Marley, M., Hubbard, W. B., et al. 1997, ApJ, 491,856

Chabrier, G., \& Baraffe, I. 2000, ARA\&A, 38, 337

Comerón, F., Rieke, G., \& Neuhäuser, R. 1999, A\&A, 343, 477 (C1999)

Comerón, F., Neuhäuser, R., \& Kaas, A. A. 2000, A\&A, 359, 269 (C2000)

Frink, S., Röser, S., Alcalá, J. M., Covino, E., \& Brandner, W. 1998, A\&A, 338, 442

Ghez, A., McCarthy, D. W., Patience, J. L., \& Beck, T. L. 1997, ApJ, 481, 378

Gizis, J. E., Kirkpatrick, J. D., Burgasser, A., et al. 2001, ApJ, 551, L163

Joergens, V., \& Guenther, E. W. 2001, A\&A, 379, L9

Kirkpatrick, J. D., Reid, I. N., Liebert, J., et al. 1999, ApJ, 519, 802 (K1999)

Kirkpatrick, J. D., Reid, I. N., Liebert, J., et al. 2000, AJ, 120, 447 (K2000)

Köhler, R. 2002, AJ, in press [astro-ph/0109103]

Koerner, D. W., Kirkpatrick, J. D., McElwain, M. W., \& Bonaventura, N. R. 1999, ApJ, 526, L25

Moffat, A. F. J. 1969, A\&A, 3, 455

Lada, C. J., Lada, E. A., Clemens, D. P., \& Bally, J. 1994, ApJ, 429, 694
Leggett, S. K., Toomey, D. W., Geballe, T. R., \& Brown, R. H. 1999, ApJ, 517, L139

Leggett, S. K., Golimowski, D. A., Fan, X., et al. 2002, ApJ, in press [astro-ph/0108435]

Lowrance, P. J., McCarthy, C., Becklin, E. E., et al. 1999, ApJ, 512, L69

Martín, E. L., Brandner, W., \& Basri, G. 1999, Science, 283, 1718

Muench, A. A., Alves, J., Lada, C. J., \& Lada, E. A. 2001, ApJ, 558, L51

Nakajima, T., Oppenheimer, B. R., Kulkarni, S. R., et al. 1995, Nature, 378, 463

Natta, A., \& Testi, L. 2001, A\&A, 376, L22

Neuhäuser, R., \& Comerón, F. 1998, Science, 282, 83

Neuhäuser, R., \& Comerón, F. 1999, A\&A, 360, 612

Neuhäuser, R., Guenther, E. W., Petr, M. G., et al. 2000, A\&A, 360, L39

Oppenheimer, B. R., Kulkarni, S. R., Matthews, K., \& van Kerkwijk, M. H. 1995, Science, 270, 1478

Persi, P., Marenzi, A. R., Olofsson, G., et al. 2000, A\&A, 357, 219

Reid, I. N., Kirkpatrick, J. D., Gizis, J. E., et al. 1999, ApJ, 521,613

Reid, I. N., Gizis, J. E., Kirkpatrick, J. D., \& Koerner, D. W. 2001, ApJ, 121, 489

Reipurth, B., \& Zinnecker, H. 1993, A\&A, 278, 81

Reipurth, B., \& Clarke, C. 2001, AJ, 122, 432

Rieke, G. H., \& Lebofsky, M. J. 1985, ApJ, 288, 618

Sterzik, M. F., Durisen, R. H., \& Pickett, B. K. 2001, Dynamical decay of young few-body clusters and the origin of isolated T Tauri stars, in Young stars near Earth, ed. R. Jayawardhana, \& T. P. Greene, ASP Conf. Ser., 244, 116

Teixeira, R., Ducourant, C., Sartori, M. J., et al. 2000, A\&A, 361,1143

Whitmore, B., Heyer, I., \& Casertano, S. 1999, PASP, 111, 1559 